\title{
Creencias y ansiedad hacia las matemáticas: un estudio comparativo entre maestros de Colombia y España
}

\author{
Beliefs and anxiety towards mathematics: a comparative study between \\ teachers of Colombia and Spain
}

\author{
Raquel Fernández-Cézar* \\ ORCID iD 0000-0002-9013-7734 \\ César Augusto Hernández-Suárez ${ }^{* *}$ \\ ORCID iD 0000-0002-7974-5560 \\ Raúl Prada-Núñez ${ }^{* * *}$ \\ ORCID iD 0000-0001-6145-1786 \\ Pastor Ramírez-Leal ${ }^{* * * *}$ \\ ORCID iD 0000-0003-3469-5325
}

\begin{abstract}
Resumen
Los estudios comparativos son interesantes para encontrar similitudes o diferencias entre contextos educativos distintos. En el ámbito Latinoamericano existen algunos sobre las actitudes del profesorado de matemáticas, pero no son muy abundantes. Con este trabajo se pretende contribuir a este cuerpo de estudios comparando dos contextos insertados en los sistemas educativos de España y Colombia. El objetivo es analizar el conjunto de creencias y actitudes hacia las Matemáticas que reportan docentes de educación básica, con especial interés sobre la ansiedad. Se partió de la validación y contextualización del cuestionario sobre creencias y actitudes hacia las matemáticas. Se siguió la metodología de la Teoría Clásica de los Test. Se aplicó una prueba piloto para calibrar
\end{abstract}

\footnotetext{
* Doctora en Ciencias Químicas de la Universidad Autónoma de Madrid (UAM). Investigadora y profesora del Departamento de Matemáticas, área de Didáctica, en la Facultad de Educación de Toledo, Universidad de Castilla La Mancha (UCLM), Castilla-La Mancha, España. Dirección postal: Despacho 1.35 Edificio Sabatini, Campus Real Fábrica de Armas, Universidad de Castilla La Mancha, 45071, Toledo, España. E-mail: Raquel.FCezar@uclm.es.

** Magister en Enseñanza de las Ciencias mención Matemática de la Universidad Nacional Experimental del Táchira (UNET). Docente e Investigador del Departamento de Pedagogia, Andragogia, Comunicación y Multimedios, en la Facultad de Educación, Artes y Humanidades de la Universidad Francisco de Paula Santander (UFPS), Cúcuta, Norte de Santander, Colombia. Dirección postal: Edificio CREAD, Universidad Francisco de Paula Santander, Av. Gran Colombia, 12E-96, Barrio Colsag, Cúcuta, Colombia, 540003. E-mail: cesaraugusto@ufps.edu.co.

*** Magister en Matemáticas mención Educación Matemática de la Universidad Nacional Experimental del Tachira (UNET). Docente e Investigador del Departamento de Ciencias Humanas, Sociales, e Idiomas, en la Facultad de Educación, Artes y Humanidades de la Universidad Francisco de Paula Santander (UFPS), Cúcuta, Norte de Santander, Colombia. Dirección postal: Edificio CREAD, Universidad Francisco de Paula Santander, Av. Gran Colombia, 12E-96 Barrio Colsag, Cúcuta, Colombia, 540003. E-mail: raulprada@ufps.edu.co.

**** Magister en Matemáticas mención Educación de la Universidad Nacional Experimental del Táchira (UNET). Docente e Investigador del Departamento de Matemáticas y Estadística, en la Facultad de Ciencias Básica de la Universidad Francisco de Paula Santander (UFPS), Cúcuta, Norte de Santander, Colombia. Dirección postal: Edificio Fundadores, Universidad Francisco de Paula Santander, Av. Gran Colombia, 12E-96 Barrio Colsag, Cúcuta, Colombia, 540003. E-mail: pastorramirez@ufps.edu.co.
} 
el instrumento; luego se administró a una muestra de 232 docentes en ambos países. Con los datos obtenidos se realizó un análisis comparativo para identificar semejanzas y diferencias entre los grupos. Los resultados evidencian similitudes epistemológicas en cuanto a los ítems relacionados con el euclidianismo. Sin embargo, en cuanto al cuasi-empirismo y el constructivismo se presentan marcadas diferencias por país. En relación con la ansiedad, las respuestas de los docentes divergen, dependiendo del contexto geográfico. Destaca el mayor nivel de ansiedad de los docentes españoles en comparación con los colombianos. Se probó que factores como el nivel de estudios y el tipo de contratación determina su nivel de ansiedad y su sistema de creencias. A futuro convendría comprobar si los niveles de ansiedad afectan, también, a la práctica docente durante la enseñanza de las matemáticas.

Palabras clave: Maestros. Educación básica. Creencias hacia las matemáticas. Posturas epistémicas. Ansiedad hacia las matemáticas. Análisis comparativo.

\begin{abstract}
Comparative studies are an interesting way to find similarities or differences between different educational contexts. In Latin America, there are some studies about the attitudes of math teachers, but they are not very abundant. This work is intended to contribute to this body of studies by comparing two contexts inserted in the educational systems of Spain and Colombia. The objective is to analyze the set of beliefs and attitudes towards Mathematics, that teachers of basic education report, with special interest on anxiety. It is based on the validation and contextualization of the questionnaire on beliefs and attitudes towards mathematics. The methodology of the Classical Test Theory was followed. A pilot test was applied to calibrate the instrument; It was then administered to a sample of 232 teachers in both countries. With the data obtained, a comparative analysis was carried out to identify similarities and differences between the groups. The results show epistemological similarities regarding the items related to Euclideanism. However, in terms of quasi-empiricism and constructivism, there are marked differences by country. In relation to anxiety, teachers' responses differ depending on the geographical context. It highlights the higher level of anxiety of Spanish teachers compared to Colombians. It was proved that factors such as the educational level and recruitment type determine your anxiety level and your belief system. In the future, it would be useful to verify whether anxiety levels also affect teaching practice during the teaching of mathematics.
\end{abstract}

Keywords: Teachers. Elementary education. Beliefs towards mathematics. Epistemic positions. Anxiety towards mathematics. Comparative analysis.

\title{
1 Introducción
}

En las últimas décadas se ha incrementado el interés por el estudio del dominio afectivo de los estudiantes y docentes universitarios en relación con las matemáticas. Esto se manifiesta en que exista un extenso apartado en la Enciclopedia de Educación Matemática de 2020 (LERMAN, 2020), así como un grupo específico (TWG08) en uno de los más prestigiosos congresos internacionales en Educación Matemática, el Congresso de la Sociedad Europea de Educación Matemática o CERME (Congres of the European Society for the Reseach in Mathematics Education) (JANKVIST; VAN DEN HEUVEL-PANHUIZEN; VELDHUIS, 2019).

En el trabajo de Ingram et al. (2020) se recogen gran parte de los estudios realizados sobre dominio afectivo. Este atractivo se debe a que la relación que guardan las actitudes, creencias y emociones con la enseñanza y el aprendizaje de las matemáticas es tan estrecha que puede llegar a generar sentimientos de miedo e inseguridad, ansiedad y bajo autoconcepto en 
las personas, lo cual puede condicionar su rendimiento en esta materia. Por otra parte, si quienes albergan estos sentimientos son los mismos docentes de matemáticas, muy probablemente los transmitirán a los alumnos desde las primeras etapas educativas, incidiendo en la reproducción de esas sensaciones negativas hacia la materia entre las nuevas generaciones de estudiantes (FERNÁNDEZ-CÉZAR; AGUIRRE-PÉREZ, 2010a), y posiblemente condicionando sus elecciones de estudios futuras.

En el contexto iberoamericano se han estudiado las actitudes hacia las matemáticas en el alumnado universitario (CARMONA, 2004; CASIS; RICO; CASTRO, 2017; MAZMACHADO et al., 2015; MARTÍNEZ-ARTERO; NORTES, 2013). Algunos de estos estudios se han realizado, concretamente, con estudiantes de los grados de Maestro en Educación Infantil y Primaria (ESTRADA, 2007; FERNÁNDEZ-CÉZAR; AGUIRRE-PÉREZ, 2010a, 2010b; MARTÍNEZ-ARTERO; NORTES, 2014; PALACIOS et al., 2013; SÁNCHEZ; SEGOVIA; MIÑÁN, 2011).

Sin embargo, son menos abundantes en este contexto los trabajos sobre el dominio afectivo de maestros de educación básica en ejercicio (GÓMEZESCOBAR; FERNÁNDEZ, 2016; FERNANDEZ-CÉZAR; PRADA-NÚÑEZ; HERNÁNDEZ-SUÁREZ, 2020). En el contexto anglosajón, no obstante, las investigaciones sobre actitudes hacia las matemáticas en los maestros ya eran numerosas desde finales del siglo pasado. Entre ellas destaca la de Wood (1988) debido a su completitud. Otros trabajos, más actuales dentro del contexto cultural anglosajón, son los de Sayers (2007), Thiel (2010), Sweeting (2011), Fives, Lacatena y Gerard (2014). En los últimos años van apareciendo estudios en otros ámbitos geográficos, como el de Uysal y Dede (2016) en el contexto turco.

Dentro de este entorno geográfico, en los últimos años están ganando terreno los estudios comparativos entre países al respecto de este dominio afectivo en estudiantes y docentes, con el fin de explorar las similitudes y diferencias que pudieran darse entre los países iberoamerianos, por compartir estos una misma lengua y tradición educativa. Asi, Estrada, Bazán y Aparicio (2010) analizaron las actitudes hacia la estadística con una muestra compuesta por profesores españoles y peruanos; Rodrigues, Fernández-Cézar y Rosa (2017) analizaron las actitudes hacia las matemáticas de los futuros maestros en España y Portugal, focalizándose en la ansiedad; Fernández-Cézar, Prada-Núñez y Solano-Pinto (2018) analizaron las creencias de maestros en servicio en España y Colombia.

Por otro lado, Fernandez-Cézar, Prada-Núñez y Hernández-Suárez (2020), han comparado el uso de procesos matemáticos en la práctica docente entre docentes de esos mismos países. Estas comparaciones forman parte de un proyecto colaborativo, establecido 
mediante el foro IBERCIENCIA. Enmarcada en ese proyecto se encuentra la presente investigación, que está dedicada al estudio de dos componentes del dominio afectivo de los maestros respecto de las matemáticas: las creencias y la ansiedad.

Distintos trabajos previos han seguido esta motivación. Así, por ejemplo, en el trabajo de Palacios et al. (2013) con estudiantes del grado de maestro, se encontró que el componente de la actitud que más determina las creencias sobre las matemáticas y el rendimiento del alumnado es la ansiedad. Por otro lado, se justifica la importancia de la investigación sobre el dominio afectivo ya que hay estudios en el mundo anglosajón que reportan una relación importante entre las actitudes hacia las matemáticas del maestro y la efectividad y calidad de su enseñanza de la disciplina en general (AIKEN, 1976; BISHOP; NICKSON, 1983; ERNEST, 1989; JONG et al., 2015; HANNULA et al., 2016, 2019) o de contenidos concretos, como la aritmética, en particular.

Godino, Batanero y Font $(2003,2004)$ señalan que el conocimiento matemático se aprende, fundamentalmente, según el enfoque sobre la materia que tiene el profesor (PRADA; HERNÁNDEZ; RAMÍREZ, 2020), lo que involucraría su actitud y creencias. En España se ha constatado, recientemente, la influencia de las prácticas docentes en el rendimiento del alumnado de cualquier etapa (MÉNDEZ, 2015), lo mismo que la existencia de una correlación positiva entre la formación en nuevas prácticas docentes del profesorado, incluyendo las TIC, y el rendimiento de sus alumnos. En este trabajo también se confirmó que el rendimiento en matemáticas de los alumnos mejora mediante el uso de prácticas tales como los trabajos en grupos pequeños, TIC y comprobación de cuadernos de ejercicios. Otro estudio, en un entorno cultural muy diferente al español, también corrobora esa tendencia (HADLEY; DORWARD, 2011).

Las creencias forman parte, junto con las emociones y las actitudes, del dominio afectivo. Las creencias son información que el sujeto acepta como verdadera (KOBALLA; CRAWLEY, 1985), y que, según Sanders Peirce (2007, p. 38) “...guían nuestros deseos y dan forma a nuestras acciones”. Además, según Gómez (2000), están constituidas por elementos cognitivos, afectivos, evaluativos y sociales que influyen en el modo en que las personas se aproximan al conocimiento matemático, y cómo se comportan respecto a él. De hecho, las creencias son consideradas parte del conocimiento mismo, que incluye aspectos de carácter perteneciente propiamente al dominio cognitivo y otros al dominio afectivo.

En efecto, el conocimiento objetivo puede ser representado fuera del individuo y el subjetivo en su interior, pero ambas formas interaccionan entre sí (PEHKONEN; PIETILÄ, 2003) influyendo en el comportamiento. De esta forma, la actitud del sujeto se conforma sobre 
la base de sus creencias, y se manifiesta en su comportamiento; de acuerdo con Pendergast, Lieberman-Betz y Vail (2017) si un maestro se percibiera con escasa habilidad para enseñar matemáticas (creencia), desarrollaría disgusto por esa área de conocimiento (actitud), e incluso se sentiría ansioso cuando tuviera que enseñarla (ansiedad). Consecuentemente, en la medida de lo posible evitaría enseñar matemáticas (comportamiento).

A partir de lo mencionado, se puede afirmar que las creencias constituyen un esquema conceptual que permite filtrar informaciones nuevas sobre la base de las procesadas anteriormente. De este modo, las creencias cumplen la función de organizar la identidad social del individuo y permiten establecer anticipaciones y juicios acerca de la realidad. En el ámbito de la enseñanza de las matemáticas, las creencias se categorizan en torno a las creencias acerca de la matemática, acerca del sujeto aprendiz, sobre la enseñanza de la matemática, y en torno al contexto en el que la enseñanza sucede (GÓMEZ-CHACÓN, 2003).

Durante el trabajo de revisión bibliográfica realizada al respecto de la posible relación entre ansiedad hacia las matemáticas, las creencias sobre las mismas, y las prácticas docentes no se encontraron trabajos previos que la confirmen o desmientan (FERNÁNDEZ et al., 2018). Existe un trabajo realizado con estudiantes para maestros chilenos (CASIS; RICO; CASTRO, 2017) en el que se analizan la motivación, la autoconfianza y la ansiedad como descriptores de la actitud hacia las matemáticas y la visión de su enseñanza. De acuerdo con los resultados de este estudio, si bien los estudiantes no muestran valores altos de ansiedad, los ítems de más alta autoconfianza se corresponden con baja ansiedad o indiferencia. También se reconoce que mediante estrategias de enseñanza apropiadas se pueden modificar esas emociones.

En esta misma línea están los resultados hallados por Ottmar et al. (2013). Uysal y Dede (2016), por su parte, analizan la ansiedad y las creencias de estudiantes para maestro en Turquía, $\mathrm{y}$, a su vez, señalan que existen pocos estudios que las relacionen con las prácticas docentes. Entre las escasas investigaciones sobre este tema se encuentra la de Haciomeroglu (2013) en el contexto turco. El autor concluye que la ansiedad afecta a las prácticas docentes en tanto que el docente no presta atención suficiente al estudiante y sus procesos de aprendizaje debido a la falta de confianza en su propia capacidad como docente de matemáticas, lo que coincide con los hallazgos de Prada, Hernández y Ramírez (2020), pero en el contexto de educación superior colombiano.

En el ámbito español, se encuentra el trabajo de León-Mantero et al. (2020) en el que se analizan las creencias hacia las matemáticas, la ansiedad y la presencia de los procesos matemáticos en la práctica docente en profesorado en ejercicio. Estas autoras encuentran que la ansiedad en el profesorado es menor en el profesorado novel y en los que tienen dilatada 
experiencia docente; que, mayoritariamente, tienen unas creencias euclidianas, y cuasiempiristas de las matemáticas, y que esta última categoría de maestros correlaciona negativamente con la ansiedad. Esto muestra que cuando el docente percibe aplicación en la vida diaria a las matemáticas (categoría cuasi-empirista) siente menos ansiedad hacia ellas. Finalmente, en México, se tiene un estudio de caso que utiliza un enfoque de historia de vida y describe el proceso de coaching seguido por una experta en educación matemática y un docente novato para superar la ansiedad matemática, que al parecer, era debida a su falta de conocimiento matemático. Este coaching permitió mostrar al docente un camino para el alivio de las emociones negativas (GARCÍA; MARTÍNEZ, 2020).

A pesar de que los estudios encuentran resultados similares en distintos contextos culturales, es importante reseñar que los instrumentos empleados en la obtención de dichos resultados pueden resultar adecuados (o no) en función de la interpretación que de ellos hacen los sujetos estudiados, y que puede estar mediada por el contexto cultural en el que viven y se desarrollan. En este sentido, es de trascendental importancia la realización de estudios comparados, especialmente en el ámbito iberoamericano y entre países que comparten la misma lengua, dado que, a pesar de ese hecho, existen diferencias en el contexto sociocultural que pueden reflejarse en los resultados.

Es preciso detectar en cada lugar las diferencias culturales y de formación, así como las posturas epistémicas que puedan afectar al sistema de creencias hacia las matemáticas por parte de los docentes. En esa línea, existen otros precedentes de estudios comparativos sobre la actitud hacia la estadística entre maestros de países iberoamericanos, como el de Estrada, Bazán y Aparicio (2010), que comparan esta actitud en maestros españoles y peruanos, y que encuentran diferencias en el conjunto de la actitud y, también, en sus distintas componentes. Además, enfatizan la necesidad de realizar más estudios de este tipo para contribuir al conocimiento de las distintas realidades educativas y de formación de maestros en los distintos países, y su posible influencia en la enseñanza.

En aras de contribuir al conocimiento de algunas realidades educativas comparadas, se desarrolla este trabajo, como parte de un proyecto promovido por la Secretaría Técnica del Área de Cooperación Científica de la Organización de Estados Iberoamericanos para la Educación, la Ciencia y la Cultura, que lleva por título ¿Influyen las actitudes y prácticas de los docentes en matemática en las actitudes del alumnado? Un estudio comparativo en el entorno iberoamericano. Se muestran, aquí, resultados parciales correspondientes a una muestra compuesta por docentes españoles y colombianos. El primer objetivo del presente trabajo de investigación fue validar el instrumento empleado mediante la Teoría Clásica de los Test 
(PALACIOS; ARIAS; ARIAS, 2014), analizando su fiabilidad, validez y sensibilidad cultural, para después poder abordar el segundo, que consiste en explorar las posibles diferencias sobre creencias y ansiedad hacia las matemáticas de los docentes y estudiantes para maestro en España y Colombia.

Al comparar los sistemas educativos colombiano y español, se observan similitudes tales como: denominación y estructura en los niveles (infantil, primaria, secundaria, media o bachillerato y superior), el proceso de formación y vinculación de docentes y diferencias específicas en el número de grados o cursos de cada nivel de formación. Estas características de los dos sistemas se resumen en el Cuadro 1, y permitieron realizar el análisis comparativo de los dos contextos.

\begin{tabular}{|c|c|c|}
\hline Características & Colombia & España \\
\hline Marco normativo & Ley General de Educación (Ley 115, 1994) & $\begin{array}{l}\text { Ley Orgánica 2/2006 de Educación para } \\
\text { Ed. Infantil/ Ley Orgánica } 8 / 2013 \text {, de } 9 \\
\text { de diciembre, para la mejora de la } \\
\text { calidad educativa (LOMCE) }\end{array}$ \\
\hline \multirow{6}{*}{ Educación Formal } & $\begin{array}{l}\text { Educación inicial (Prejardín } 3 \text { años, Jardín } \\
4 \text { años) no obligatoria }\end{array}$ & \multirow{2}{*}{$\begin{array}{l}\text { Educación infantil, organizada en dos } \\
\text { ciclos (3-6 años), no obligatoria. }\end{array}$} \\
\hline & $\begin{array}{l}\text { Educación preescolar (en esta etapa de } \\
\text { Transición } 5 \text { años, siendo este el inicio del } \\
\text { nivel Cero obligatorio) }\end{array}$ & \\
\hline & \multirow{2}{*}{$\begin{array}{l}\text { Educación } \\
\text { Básica }\end{array}$} & $\begin{array}{l}\text { Educación primaria ( } 6 \text { cursos, de } \\
\text { primero a sexto obligatorios })\end{array}$ \\
\hline & & $\begin{array}{l}\text { Educación secundaria - ESO ( } 4 \text { cursos, } \\
\text { de primero a cuarto obligatorios) }\end{array}$ \\
\hline & $\begin{array}{l}\text { Educación media, comprende dos grados y } \\
\text { sirve de preparación para el ingreso a la } \\
\text { educación superior y al trabajo (no } \\
\text { obligatorio) }\end{array}$ & $\begin{array}{l}\text { El Bachillerato (no obligatorio) } \\
\text { comprende dos cursos y se desarrolla en } \\
\text { tres modalidades diferentes }\end{array}$ \\
\hline & $\begin{array}{l}\text { Educación Superior, a nivel técnico, } \\
\text { tecnológico y profesional }\end{array}$ & $\begin{array}{l}\text { La formación profesional comprende un } \\
\text { conjunto de ciclos formativos de grado } \\
\text { medio y de grado superior }\end{array}$ \\
\hline \multirow{6}{*}{$\begin{array}{l}\text { Formación de } \\
\text { docentes }\end{array}$} & \multicolumn{2}{|c|}{ Educación Infantil } \\
\hline & $\begin{array}{l}\text { Licenciatura en Educación Infantil o afines } \\
\text { o Normalista Superior }\end{array}$ & $\begin{array}{l}\text { Título de Grado en Educación Infantil o } \\
\text { título de Maestro }\end{array}$ \\
\hline & \multicolumn{2}{|c|}{ Educación Primaria } \\
\hline & $\begin{array}{l}\text { Licenciado en cualquier modalidad, } \\
\text { Tecnólogo en Educación o Normalista } \\
\text { Superior }\end{array}$ & $\begin{array}{l}\text { Título de Grado en Educación primaria } \\
\text { o título de Maestro }\end{array}$ \\
\hline & Básica Secundaria y/o Media & $\begin{array}{c}\text { Enseñanza Secundaria (ESO) y } \\
\text { Bachillerato }\end{array}$ \\
\hline & $\begin{array}{l}\text { Licenciado en área específica, o área afín, } \\
\text { o profesional con título diferente al de } \\
\text { Licenciado }\end{array}$ & $\begin{array}{l}\text { Título de Grado, Doctor, Licenciado, } \\
\text { Ingeniero, Arquitecto u otros títulos } \\
\text { equivalentes a efectos de docencia. } \\
\text { Además, es necesario el Máster de } \\
\text { Profesorado de Secundaria }\end{array}$ \\
\hline $\begin{array}{l}\text { Docente en } \\
\text { matemáticas }\end{array}$ & $\begin{array}{l}\text { Licenciatura en Matemáticas (solo, con } \\
\text { otra opción o con énfasis), Licenciatura en } \\
\text { Física (solo, con otra opción o con énfasis), } \\
\text { Licenciatura en educación básica con } \\
\text { énfasis en matemáticas, Título profesional } \\
\text { universitario con núcleo básico en } \\
\text { Matemáticas, Estadística, Física (solo o }\end{array}$ & $\begin{array}{l}\text { Profesorado en Matemáticas, } \\
\text { Matemático, también pueden optar } \\
\text { Químicos, Físicos, e Ingenieros. }\end{array}$ \\
\hline
\end{tabular}




\begin{tabular}{|c|l|l|l|}
\hline & $\begin{array}{l}\text { con énfasis), y cualquier título de } \\
\text { Ingeniería }\end{array}$ & \\
\hline \multirow{2}{*}{$\begin{array}{c}\text { Ejercicios de la } \\
\text { docencia }\end{array}$} & $\begin{array}{l}\text { Instituciones educativas o centros públicos } \\
\text { Concurso docente (Convocatoria Pública); } \\
\text { Docente en provisionalidad (o temporal } \\
\text { hasta que se ocupe la vacante de forma } \\
\text { definitiva) }\end{array}$ & $\begin{array}{l}\text { Oposición competitivo). } \\
\text { comen }\end{array}$ \\
\cline { 2 - 4 } & \multicolumn{3}{|c|}{ Instituciones educativas o centros privados } \\
\hline
\end{tabular}

Cuadro 1 - Comparación de las características de los Sistemas Educativos de Colombia y España Fuente: elaborado por los autores

\section{Marco conceptual}

\subsection{Creencias sobre las matemáticas}

Entre los investigadores en educación matemática, tal y como señalan Goldin, Rösken y Törner (2009), no existe una definición de creencias unánimemente aceptada. Además, es necesario reconsiderar el papel de los marcos teóricos dominantes, incluida la conceptualización de la construcción clave de las creencias y los principales métodos de investigación (SKOTT, 2014). Sin embargo, todos los trabajos las consideran como un aspecto del dominio cognitivo, influida por, e influyente en el afectivo. Así, Gómez-Chacón (2003) define las creencias como una parte del conocimiento (perteneciente, por tanto, al dominio cognitivo), pero que también está compuesta por elementos afectivos, evaluativos y sociales. De esta manera, mediante sus creencias el individuo se posiciona respecto al conocimiento matemático, a qué es y a cómo se aborda, haciendo una interpretación social del mismo. Esta interpretación de las creencias es la que se asume en este trabajo.

Sin embargo, los constructos a través de los cuales se miden o cuantifican las creencias suelen coincidir en que estas son representaciones internas a las cuales el poseedor les atribuye verdad, validez o aplicabilidad. Usualmente son estables, altamente cognitivas y pueden estar fuertemente estructuradas (REYES-RODRÍGUEZ et al., 2017). En este sentido, las creencias son independientes de su validez social, por lo que, desde una perspectiva epistemológica, las creencias son constructos individuales, mientras que el conocimiento es un constructo social (OP'T EYNDE; DE CORTE; VERSCHAFFEL, 2003).

Ramos (2005), afirma que la investigación sobre las concepciones y creencias del profesorado de matemáticas muestra que todo cambio que se pretenda introducir en las instituciones escolares no puede prescindir del conocimiento y, tal vez, de una modificación de 
las opiniones del profesorado sobre qué son las matemáticas, puesto que dicha opinión incide sobre cómo se enseñan las matemáticas.

Además, Thompson (1992, p. 132) afirma, sobre las concepciones de la naturaleza de las matemáticas por parte de los maestros, lo siguiente:

\begin{abstract}
La concepción de un maestro sobre la naturaleza de las matemáticas puede verse como las creencias conceptos, significados, reglas, imágenes mentales y preferencias conscientes e inconscientes con respecto a la disciplina de las matemáticas. Esas creencias, conceptos, puntos de vista, y preferencias constituyen los rudimentos de una filosofía de las matemáticas, aunque para algunos maestros pueden no desarrollado y articulados en una filosofía coherente ${ }^{1}$ (Nuestra traducción).
\end{abstract}

Por otra parte, Gascón (2001), señala que las decisiones y actuaciones docentes e incluso, hasta ciertos modelos docentes, están relativamente estructurados con los modelos epistemológicos generales que han existido a lo largo de la historia de las matemáticas y que, en cierta forma, perviven entremezclados en las diferentes instituciones didácticas. Es decir, que es casi imposible separar la construcción social de la institución del conocimiento matemático. Según Lakatos (1978) en la organización de las matemáticas se pueden distinguir dos teorías epistemológicas generales: teorías euclídeas y teorías cuasi-empíricas. Para completar este esquema (GASCÓN, 2001) añade un tercer grupo de teorías epistemológicas, llamadas teorías constructivistas (ver Cuadro 2).

\begin{tabular}{|c|c|c|c|}
\hline \multicolumn{2}{|c|}{ Modelo epistemológico } & $\begin{array}{l}\text { Forma de enseñar } \\
\text { Gascón }(2001)\end{array}$ & Autores \\
\hline \multirow{3}{*}{ 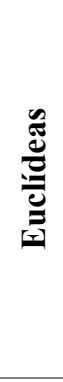 } & Concepto & $\begin{array}{l}\text { El proceso de enseñanza es mecánico y } \\
\text { trivial, totalmente controlable por el } \\
\text { docente. }\end{array}$ & \multirow{3}{*}{$\begin{array}{l}\text { Las matemáticas se conciben como un } \\
\text { cuerpo inamovible de conocimientos, } \\
\text { autónomo de la realidad. }\end{array}$} \\
\hline & Teoricismo & $\begin{array}{l}\text { Hace énfasis en la enseñanza de las } \\
\text { teorías, el docente se enfoca en la } \\
\text { enseñanza del teorema o concepto. }\end{array}$ & \\
\hline & Tecnicismo & $\begin{array}{l}\text { La enseñanza se enfoca en aprender, } \\
\text { mediante repetición, diversas técnicas } \\
\text { algorítmicas. }\end{array}$ & \\
\hline \multirow{2}{*}{ 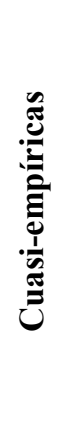 } & Concepto & $\begin{array}{l}\text { Es una teoría en formación donde lo } \\
\text { esencial son los procedimientos (no } \\
\text { algorítmicos), donde se busca } \\
\text { conjeturar, probar, contrastar, refutar, } \\
\text { buscar contractar ejemplos, cambiar un } \\
\text { poco el problema original y nunca } \\
\text { llegando a una conclusión verdadera. }\end{array}$ & \multirow{2}{*}{$\begin{array}{l}\text { Las matemáticas se conciben como un } \\
\text { cuerpo de conocimientos derivados del } \\
\text { intento de conocer e interpretar el } \\
\text { entorno que nos rodea, a la vez que son } \\
\text { útiles para ello. }\end{array}$} \\
\hline & Modernismo & $\begin{array}{l}\text { Considera el proceso de aprendizaje } \\
\text { como un proceso deductivo, intuitivo y } \\
\text { autónomo, totalmente controlado por el } \\
\text { profesor. }\end{array}$ & \\
\hline
\end{tabular}

\footnotetext{
${ }^{1}$ A teacher's conception of the nature of mathematics may be viewed as that teacher's conscious or subconscious beliefs, concepts, meanings, rules, mental images, and preferences concerning the discipline of mathematics. Those beliefs, concepts, view, and preferences constitute the rudiments of a philosophy of mathematics, although for some teachers they may not be developed and articulated into a coherent philosophy.
} 


\begin{tabular}{|c|c|c|c|}
\hline & Procedimentalismo & $\begin{array}{l}\text { Se enseña mediante un proceso } \\
\text { didáctico y estructurado de técnicas } \\
\text { heurísticas no algorítmicas. }\end{array}$ & \\
\hline 党 & Concepto & $\begin{array}{l}\text { Percibe el aprendizaje de la matemática } \\
\text { como la construcción de conocimiento } \\
\text { a través de modelos matemáticos } \\
\text { extraídos de los conceptos manejados } \\
\text { en el aula, es decir, genera el } \\
\text { conocimiento con el sistema que se está } \\
\text { modelando, para lo cual requiere de } \\
\text { problemas contextualizados. }\end{array}$ & $\begin{array}{l}\text { Las matemáticas se conciben como un } \\
\text { cuerpo de conocimiento que se basan } \\
\text { en otros conocimientos previos, y que } \\
\text { se construye a partir de la realidad de } \\
\text { cada aprendiz. }\end{array}$ \\
\hline
\end{tabular}

Cuadro 2 - Posturas epistemológicas

Fuente: elaborado por los autores

Son particularmente interesantes los estudios de las creencias sobre las matemáticas de los docentes o maestros por su reflejo, o posible influencia, en los discentes (LEÓNMANTERO et al., 2020). Sin embargo, son más abundantes las investigaciones que abordan las creencias de los docentes sobre algún dominio concreto de las matemáticas con una perspectiva instrumental. Entre ellos, destacan trabajos sobre resolución de problemas (ANDREWS; XENOFONTOS, 2015; LERA; DEULOFEU, 2014, 2015; SIVUNEN; PEHKONEN, 2009; VILA; CALLEJO, 2004); sobre el concepto de función (LLINARES, 1996); o sobre la enseñanza de la Geometría (PÉREZ; GUILLÉN, 2007).

Según Hannula et al. (2016), los estudios acerca de las creencias sobre las matemáticas de los docentes se vienen desarrollando en dos líneas principales: las investigaciones sobre la relación entre las creencias y la práctica docente y las investigaciones sobre las evoluciones o los cambios en las creencias de los docentes. La primera es de particular interés en este estudio. En la revisión bibliográfica realizada se detecta que los resultados de las investigaciones orientadas al establecimiento de la relación entre creencias y prácticas docentes han sido muy dispares. Aun así, se ha establecido una serie de factores que pueden influir en las creencias. Entre ellos se encuentra, además del conocimiento, la experiencia personal y el contexto de los docentes, pero deben ampliarse las investigaciones orientadas a confirmar, o no, su importancia.

Por esta razón, en este estudio se ha decidido profundizar en la influencia de la sensibilidad cultural en la constitución de las creencias del sujeto, para lo cual se ha construido un instrumento a partir del cuestionario de creencias de Godino, Batanero y Font $(2003,2004)$ que fue elaborado a partir del libro de Baroody y Coslick (1998) y empleado por León-Mantero et al. (2020). 


\subsection{Ansiedad hacia las matemáticas}

La importancia de la asignatura de matemáticas ha sido valorada de diferentes maneras, yendo desde su consideración como asignatura destacada para medir el rendimiento académico hasta su carácter predictivo del cociente intelectual. Precisamente por estas asociaciones, las matemáticas son susceptibles de ser consideradas difíciles y su estudio se relaciona con una ansiedad excesiva tanto en el alumnado como en el profesorado (MARTíNEZ, 2005) y las familias (RODRÍGUEZ-MANTILLA; FERNÁNDEZ-CRUZ, 2017).

En el concepto de ansiedad se ha dado importancia a las situaciones que la elicitan, con énfasis en la interpretación que la persona realiza de tales situaciones y la anticipación que hace de posibles aspectos amenazantes. Algunos autores destacan que la ansiedad es una actitud emocional cognitiva, básica, universal y adaptativa que acompaña al ser humano y que provoca la activación del organismo como respuesta ante situaciones percibidas como amenazantes (FERNÁNDEZ-ABASCAL; JIMÉNEZ; MARTÍN, 2003). Pero, también se les considera como un conjunto de emociones negativas (GARCÍA; MARTÍNEZ, 2020).

En circunstancias normales, y en un grado controlable, la ansiedad forma parte de la experiencia que permite a la persona afrontar las demandas de las tareas propias de su proceso de aprendizaje. Ahora bien, si los niveles de ansiedad son excesivos pueden limitar, bloquear e, incluso, hacer que la persona evite afrontar cualquier aspecto que interprete como amenazante asociado al estudio, en este caso, de las matemáticas. En este sentido, si las situaciones de aprendizaje de las matemáticas son percibidas e interpretadas como circunstancias amenazantes, el sujeto puede llegar a evitarlas o evadirse de los contextos en los que su uso es necesario.

En el caso de los maestros, las consecuencias son, todavía, más severas, pues si el docente se siente evaluado, siente que su alumnado percibirá sus limitaciones como profesor, si además anticipa que él no es capaz de reflexionar sobre determinados supuestos matemáticos, aprender o enseñar matemáticas se puede convertir en un camino tortuoso en el que incluso se pueden ver mermadas sus capacidades cognitivas (DOGAN, 2012).

La ansiedad ante las matemáticas se caracteriza por un intenso e irracional malestar ante situaciones relacionadas con ellas, que se englobará en las manifestaciones de los tres sistemas de respuesta: cognitivo, fisiológico y motor (PÉREZ-TYTECA et al., 2011). Esta es la conceptualización que se asume en este trabajo: la ausencia de confort o malestar al respecto de las matemáticas experimentado por el profesorado influirá en su actuación al respecto de enseñanza de estas. 
Según los estudios de Sánchez, Segovia y Miñán (2011), los niveles altos de ansiedad se encuentran presentes entre el alumnado de Magisterio, estadio previo al de los maestros de Educación Elemental, equivalente a la Educación Primaria. Wood (1988), por su parte, reporta que el $16 \%$ de estos maestros pueden clasificarse como personas con ansiedad hacia las matemáticas, $\mathrm{y}$, a veces, los temores son tan elevados que necesitan ayuda y acompañamiento para superarlos. Por lo tanto, la importancia de conocer si los maestros sienten ansiedad hacia las matemáticas es de gran importancia, sobre todo de cara a las posibles consecuencias que manifestarían en su enseñanza. Además, ¿cómo podría un maestro enseñar bien matemáticas si no se siente cómodo en esa asignatura?

Así pues, resulta esencial tener un diagnóstico de la situación entre los maestros al respecto de la ansiedad hacia las matemáticas, pues sería muy difícil conseguir que los alumnos adultos eligieran clases de matemáticas en sus estudios futuros si actualmente sus maestros adolecieran de matemafobia (en inglés mathophobia, término acuñado por Mihalko (1978 apud WOOD, 1988). El sentimiento negativo hacia las matemáticas podría hacer que el profesorado fuera más rígido y controlador en el proceso de enseñanza- aprendizaje, lo que originaría mayores niveles de ansiedad entre sus estudiantes (ASSOR et al., 2005).

Este sentimiento también tendría influencia en las prácticas docentes, puesto que puede abocar al profesor a adoptar estrategias de enseñanza centradas en los contenidos en lugar de en el alumnado, con el objetivo de disminuir su malestar emocional a través del control. Esto se constata en trabajos realizados en diferentes entornos socioculturales, como el de Brígido et al. (2010), el de Uysal y Dede (2016), de carácter cuantitativo, o el de García y Martínez (2020) de carácter cualitativo.

Sobre los cuestionarios empleados para estudiar la ansiedad, el más ampliamente difundido es el Mathematics Anxiety Research Scale (MARS), del que fue publicada una versión abreviada con validación psicométrica por Suinn y Winston (2003). En el ámbito hispanoamericano, el Cuestionario de Actitudes hacia las Matemáticas, de Auzmendi (1992), es el más ampliamente empleado. Este cuestionario ha sido aplicado a maestros de educación infantil y primaria (FERNÁNDEZ-CÉZAR et al., 2016), y, mediante análisis factorial exploratorio y confirmatorio, se concluye que es un instrumento con propiedades psicométricas aceptables para la medida de la ansiedad. La versión que aquí se ha empleado se denomina Cuestionario de Auzmendi Modificado. 


\section{Metodología}

A fin de satisfacer el propósito general, este estudio comparativo se plantea como una investigación cuantitativa, de naturaleza descriptiva y correlacional, pues a partir de los datos colectados se busca recabar evidencias acerca de las similitudes y diferencias entre el conjunto de creencias y la ansiedad manifestada por docentes españoles y colombianos hacía las matemáticas.

Se considera también importante analizar la posible influencia de los factores personales y socioeducativos que describen la muestra, en línea con el realizado con maestros españoles por León-Mantero et al. (2020), en tanto que podrían afectar en alguna manera a las creencias sobre las matemáticas y la ansiedad de los docentes hacia estas; estos factores pueden afectar la ansiedad general de los participantes, y en particular, su ansiedad hacia las matemáticas. Sin embargo, las creencias hacia las matemáticas pueden no ser susceptibles de afectación por estos, en tanto que son construcciones más personales e individuales. A continuación, se describen las características principales del proceso metodológico seguido para la recolección de los datos.

\subsection{Muestra}

La muestra es de conveniencia y está constituida por las respuestas de los docentes que accedieron a completar el cuestionario, que ascienden a 232, de los cuales 105 son españoles (45.4\%) y 127 colombianos (54.6\%). En el caso de España, el cuestionario se difundió en línea y fue cumplimentado por docentes, graduados o diplomados en ejercicio, y por estudiantes para maestro o futuros docentes. En el caso de Colombia, se administró en versión impresa. Participaron tres grupos de informantes: docentes, docentes en formación y otros (mentores, asesores o tutores). Las características principales de la muestra se relacionan en las Tablas 1 y 2. La proporción de estudiantes no titulados es baja, suponiendo menos del $4 \%$ del conjunto. De igual manera, menos del 1\% de la muestra corresponde a asesores o mentores.

Tabla 1 - Tipo de participantes por país

\begin{tabular}{cccccccc}
\hline & & \multicolumn{2}{c}{ España } & \multicolumn{2}{c}{ Colombia } & \multicolumn{2}{c}{ Total } \\
\cline { 2 - 8 } & & $\mathrm{F}$ & $\%$ & $\mathrm{~F}$ & $\%$ & $\mathrm{~F}$ & $\%$ \\
\hline \multirow{3}{*}{ Tipo de Participante } & Docente & 95 & $40.9 \%$ & 125 & $53.9 \%$ & 220 & $94.8 \%$ \\
\cline { 2 - 7 } & Docentes en formación & 8 & $3.4 \%$ & 0 & $0.0 \%$ & 8 & $3.4 \%$ \\
\cline { 2 - 7 } & Otro & 2 & $0.9 \%$ & 2 & $0.9 \%$ & 4 & $1.7 \%$ \\
\cline { 2 - 7 } & Total & 105 & $45.3 \%$ & 127 & $54.7 \%$ & 232 & $100.0 \%$ \\
\hline & Fuente: elaborada por los autores & & &
\end{tabular}

La Tabla 2 está desagregado por país, diversas características de los docentes y su 
porcentaje sobre el tamaño total de la muestra. Se observa que la distribución por tipo de contratación es equiparable en ambos países, alcanzando los contratos fijos aproximadamente el $30.2 \%$ de la muestra de España y el $32.7 \%$ de la muestra de Colombia, mientras que se encuentra una diferencia algo mayor en los contratos temporales, $10.8 \%$ y $16.4 \%$, respectivamente. El $25.9 \%$ de los docentes colombianos tienen grados de Licenciatura y el $22.4 \%$ ha cursado estudios de postgrado. Por otro lado, en el caso español, menos del $2 \%$ estudia postgrado. Finalmente, se observa que en ambos países el porcentaje de docentes integrantes de la muestra, que trabajan en instituciones educativas públicas es similar.

Tabla 2 - Perfil de los participantes

\begin{tabular}{|c|c|c|c|c|c|}
\hline & & \multicolumn{4}{|c|}{ País } \\
\hline & & \multicolumn{2}{|c|}{ España } & \multicolumn{2}{|c|}{ Colombia } \\
\hline & & $\mathrm{F}$ & $\%$ & F & $\%$ \\
\hline \multirow{4}{*}{ Tipo de Contrato } & Desempleado/Otro & 10 & $4.3 \%$ & 13 & $5.6 \%$ \\
\hline & Contrato temporal & 25 & $10.8 \%$ & 38 & $16.4 \%$ \\
\hline & Contrato permanente & 70 & $30.2 \%$ & 76 & $32.7 \%$ \\
\hline & Total & 105 & $45.3 \%$ & 127 & $54.7 \%$ \\
\hline \multirow{5}{*}{ Tipo de Institución } & No trabaja & 5 & $2.2 \%$ & 0 & $0.0 \%$ \\
\hline & Público & 83 & $35.8 \%$ & 82 & $35.4 \%$ \\
\hline & Concertado & 13 & $5.6 \%$ & 37 & $15.9 \%$ \\
\hline & Otro & 4 & $1.7 \%$ & 8 & $3.4 \%$ \\
\hline & Total & 105 & $45.3 \%$ & 127 & $54.7 \%$ \\
\hline \multirow{6}{*}{ Nivel de estudios } & Diplomatura & 50 & $21.6 \%$ & 7 & $3.0 \%$ \\
\hline & Licenciatura & 35 & $15.1 \%$ & 60 & $25.9 \%$ \\
\hline & Grado & 16 & $6.9 \%$ & 0 & $0.0 \%$ \\
\hline & Post Grado (Maestría/Doctorado) & 4 & $1.7 \%$ & 52 & $22.4 \%$ \\
\hline & Otro & 0 & $0.0 \%$ & 8 & $3.4 \%$ \\
\hline & Total & 105 & $45.3 \%$ & 127 & $54.7 \%$ \\
\hline
\end{tabular}

\subsection{Instrumento de medición: validez y confiabilidad}

El cuestionario sobre creencias y actitudes, elaborado para este estudio en el contexto colombiano y el español, se realiza a partir del cuestionario de creencias de Godino, Batanero y Font (2003, 2004), a su vez elaborado a partir del libro de Baroody y Coslick (1998), para determinar si el profesor tiene una concepción de las matemáticas euclidiana, cuasi-empirista o constructivista (GARCÍA-MOYA et al., 2020; LEÓN-MANTERO et al., 2020). Por otro lado, se toma el cuestionario de actitudes hacia las matemáticas de Auzmendi (1992) modificado, empleado por Fernández-Cézar et al. (2016). En ambos la respuesta se propone como escala tipo Likert de 1 a 5, donde 1 es totalmente en desacuerdo y 5 totalmente de acuerdo.

Se toma este instrumento porque, en este trabajo, al igual que Auzmendi (1992) se considera la ansiedad como componente de la actitud. También lo hacen otros autores como Casis, Rico y Castro, (2017) y Fernández-Abascal, Jiménez y Martín (2003), que consideran la 
ansiedad como un descriptor de las actitudes hacia las matemáticas específicamente como actitud emocional cognitiva, básica, universal y adaptativa que acompaña al ser humano y que provoca la activación del organismo como respuesta ante situaciones percibidas como amenazantes.

\subsection{Análisis de los datos}

Puesto que se trata de un estudio comparativo, se realizó, en primer lugar, un análisis descriptivo para analizar las tendencias en las respuestas. Para ello, se dispuso una tabla de distribución de frecuencias por conjuntos. Las opciones de respuesta de totalmente de acuerdo y de acuerdo se agruparon en una categoría mayor, que se denominó acuerdo. De la misma manera, las opciones de respuesta totalmente en desacuerdo y en desacuerdo, se agruparon en una sola categoría llamada desacuerdo.

En segundo lugar, se evaluó la significatividad de las diferencias considerando como variables dependientes las puntuaciones totales de las escalas de creencias y ansiedad, y como factores las diferentes características del perfil de los docentes. Para ello, se analizó la normalidad de la distribución de los valores de creencias y ansiedad obteniendo valores de p>.05 con la prueba Kolgomorov-Smirnov, ratificando la normalidad de los datos, y se ejecutó la comparación de medias a través del ANOVA de un factor y, en caso de encontrar diferencias significativas, las pruebas post hoc de Duncan. Todos los contrastes se realizaron con un nivel .05 de significación y se empleó el paquete informático SPSS versión 23.0. La correlación se analizó mediante el coeficiente $r$ de Pearson, al considerar las variables tratadas como numéricas.

\section{Resultados}

Se realizó la validación del cuestionario empleado en este trabajo en primer lugar. Para cada caso se tomó una muestra piloto de cuarenta informantes, con el fin de realizar un análisis de los ítems bajo los parámetros de la Teoría Clásica de los Test (TCT), cuyo enfoque consiste en asegurarse de que el instrumento que se obtiene mida con la mayor precisión y el mínimo error aquello que se quiere medir. La teoría clásica sugiere el análisis de la validez, confiabilidad y sensibilidad cultural (COHEN; SWERDLKY, 2010).

El concepto de validez de un instrumento es muy amplio y supone varias etapas. La consulta a expertos es la vía más usual para apreciar la calidad del contenido, especialmente en 
ámbitos educativos, aunque cada vez son más empleados los métodos cualitativos basados en la observación directa, las entrevistas o el análisis de archivos (PRIETO; DELGADO, 2010; SIRECI, 1998). En este caso, se empleó el juicio de expertos, que incluyó varias rondas con especialistas en el área, cuyas observaciones se fueron incorporando hasta alcanzar un consenso en el diseño y redacción de los ítems. Estos quedaron redactados tal y como se muestran en la Tabla 3.

Tabla 3 - Índices de discriminación: correlación Ítem - Total aplicando la TCT para cada ítem del cuestionario

\begin{tabular}{|c|c|c|c|c|c|}
\hline & Ítem & $\begin{array}{c}\text { Media de } \\
\text { escala si el } \\
\text { elemento se } \\
\text { ha suprimido }\end{array}$ & $\begin{array}{l}\text { Varianza de } \\
\text { escala si el } \\
\text { elemento se } \\
\text { ha suprimido }\end{array}$ & $\begin{array}{l}\text { Correlación } \\
\text { total de } \\
\text { elementos } \\
\text { corregida }\end{array}$ & $\begin{array}{c}\text { Alfa de } \\
\text { Cronbach si } \\
\text { el elemento se } \\
\text { ha suprimido }\end{array}$ \\
\hline P1_1 & $\begin{array}{lr}\text { Las matemáticas } & \text { son } \\
\text { esencialmente un sistema } \\
\text { complejo de conceptos, } \\
\text { procedimientos } & y \\
\text { representaciones } & \\
\text { interconectadas. } & \\
\end{array}$ & 187.14 & 933.980 & .533 & .952 \\
\hline P1_2 & $\begin{array}{l}\text { Las matemáticas son un } \\
\text { cuerpo de conocimiento } \\
\text { estructurado lógicamente. }\end{array}$ & 186.45 & 956.328 & $.344(*)$ & .953 \\
\hline P1_3 & $\begin{array}{l}\text { Para mí las matemáticas son } \\
\text { un cuerpo de conocimientos } \\
\text { objetivo, absoluto, universal, } \\
\text { libre de valores y abstracto. }\end{array}$ & 187.24 & 943.761 & $.393(*)$ & .953 \\
\hline P1_4 & $\begin{array}{lr}\text { Las matemáticas implican } \\
\text { principalmente } \\
\text { memorización } \\
\text { procedimientos } \\
\text { seguimiento de reglas. }\end{array}$ & 187.72 & 953.778 & $.200(*)$ & .954 \\
\hline P1_5 & $\begin{array}{l}\text { El dominio de las } \\
\text { matemáticas se caracteriza } \\
\text { por la aplicación de reglas y } \\
\text { algoritmos. }\end{array}$ & 187.24 & 954.690 & $.187(*)$ & .954 \\
\hline P1_6 & $\begin{array}{l}\text { El conocimiento matemático } \\
\text { esencialmente es fijo e } \\
\text { inmutable. }\end{array}$ & 187.66 & 949.805 & $.287(*)$ & .953 \\
\hline P1_7 & $\begin{array}{lr}\text { Las matemáticas están } \\
\text { abiertas a cuestionamientos, } \\
\text { argumentos } \\
\text { interpretaciones personales } \\
\text { relativas al contexto. }\end{array}$ & 186.55 & 957.756 & $.223(*)$ & .953 \\
\hline P1_8 & $\begin{array}{l}\text { La habilidad matemática es } \\
\text { esencialmente algo con lo } \\
\text { que se nace o no se nace. }\end{array}$ & 188.10 & 956.453 & $.159(*)$ & .954 \\
\hline P1_9 & $\begin{array}{l}\text { El dominio de las } \\
\text { matemáticas se materializa } \\
\text { en una manera de pensar y } \\
\text { resolver problemas. }\end{array}$ & 186.72 & 955.850 & $.227(*)$ & .953 \\
\hline P2_1 & $\begin{array}{l}\text { Las matemáticas es la } \\
\text { asignatura que más me gusta } \\
\text { enseñar. }\end{array}$ & 186.45 & 958.828 & $.209(*)$ & .953 \\
\hline
\end{tabular}




\begin{tabular}{|c|c|c|c|c|c|}
\hline P2_2 & $\begin{array}{l}\text { Las matemáticas han sido } \\
\text { una asignatura difícil para } \\
\text { mí. }\end{array}$ & 187.97 & 963.749 & $.037(*)$ & .955 \\
\hline $\mathrm{P} 2 \_3$ & $\begin{array}{l}\text { Las matemáticas son una } \\
\text { asignatura que parece difícil } \\
\text { a mis alumnos. }\end{array}$ & 187.10 & 961.596 & $.077(*)$ & .954 \\
\hline $\mathrm{P} 2 \_4$ & $\begin{array}{l}\text { Las matemáticas eran/son } \\
\text { una de las asignaturas que } \\
\text { más temía/temo. }\end{array}$ & 187.48 & 964.687 & $.027(*)$ & .955 \\
\hline P2_5 & $\begin{array}{l}\text { Tengo confianza en mí } \\
\text { cuando me enfrento a un } \\
\text { problema de matemáticas. }\end{array}$ & 186.38 & 957.530 & $.335(*)$ & .953 \\
\hline P2_6 & $\begin{array}{l}\text { Cuando me enfrento a un } \\
\text { problema de matemáticas me } \\
\text { siento incapaz de pensar con } \\
\text { claridad. }\end{array}$ & 188.34 & 977.377 & $-.171(*)$ & .955 \\
\hline $\mathrm{P} 2 \_7$ & $\begin{array}{l}\text { Estoy calmado/a } \quad \text { y } \\
\text { tranquilo/a cuando me } \\
\text { enfrento a un problema de } \\
\text { matemáticas. }\end{array}$ & 186.52 & 963.973 & .083 & .954 \\
\hline P2_8 & $\begin{array}{l}\text { Trabajar con las matemáticas } \\
\text { hace que me sienta muy } \\
\text { nervioso/a. }\end{array}$ & 188.38 & 971.101 & -.086 & .955 \\
\hline P2_9 & $\begin{array}{l}\text { Me altero cuando tengo que } \\
\text { trabajar en problemas de } \\
\text { matemáticas. }\end{array}$ & 188.24 & 961.904 & .075 & .954 \\
\hline P2_10 & $\begin{array}{l}\text { Las matemáticas hacen que } \\
\text { me sienta incómodo/a y } \\
\text { nervioso/a. }\end{array}$ & 188.45 & 963.113 & $.076(*)$ & .954 \\
\hline
\end{tabular}

Fuente: elaborada por los autores

Para el proceso de validación se trabajó en esta secuencia: en primer lugar, se determinó la capacidad discriminativa de cada ítem y se reportó su aporte a cada subescala y a la escala global; en segundo lugar, se propusieron sugerencias para mejorar la validez y confiabilidad de cada uno. Otro aspecto para considerar en el cuestionario fue la sensibilidad cultural. Su medición se basa en el estudio de los ítems con referencia a su imparcialidad en relación con los posibles examinados, a fin de evitar el uso de lenguaje ofensivo, estereotipos o situaciones adversas (COHEN; SWERDLIK, 2010). Este indicador fue trabajado a partir del juicio de expertos y con la revisión por parte del personal al cual iba dirigido el test, considerando sus modificaciones.

La fiabilidad del instrumento fue abordada desde la Teoría Clásica de los Test, analizando, principalmente, su índice de discriminación. Dado que los ítems del instrumento corresponden a variables no cognitivas, el principal indicador para evaluar el comportamiento individual de cada ítem y su contribución a cada subescala y la escala global es su capacidad discriminativa.

Dada la estructura del cuestionario y la evaluación de los tiempos de aplicación de la prueba, se tomó como criterio de confiabilidad el concepto de consistencia interna de los ítems, 
y por tratarse de escalas polinómicas, el coeficiente de confiabilidad alfa de Cronbach es el método que se considera más adecuado. De acuerdo con Muñiz et al. (2005), un ítem discrimina muy bien si su índice de discriminación (Id) es al menos .40. Si se encuentra en el rango entre .30 y .39 discrimina bien, y si está entre .10 y .29 se debe revisar. Valores inferiores cuestionan la capacidad discriminativa del ítem.

Para la muestra de la prueba piloto se sugirió la revisión de algunos ítems, ya que su capacidad discriminativa resultó comprometida $(\mathrm{Id}<.30)$. En consecuencia, se replanteó su formulación y luego se incorporaron a la versión final del instrumento. Los valores de los índices de discriminación de la totalidad de los ítems a escala global oscilan entre -.07 y .87. Aunque el valor promedio de los índices de discriminación es bastante alto (próximo a .60), se observó que algunos ítems se hallaban fuera del rango válido sugerido para reflejar una adecuada capacidad de discriminación. Estos ítems son principalmente de la escala 1, referida a las creencias, desde el ítem identificado como P1_4 hasta el P1_9.

Similarmente, para la escala 2 de ansiedad, los ítems que requirieron una reelaboración fueron las que van desde el P2_1 al P2_5, y el P2_10 (ver Tabla 3). Estos ítems fueron revisados en su redacción y reformulados para la aplicación en su versión definitiva a la muestra colombiana considerada. Una vez analizados los ítems e incorporados los cambios, el valor del alfa de Cronbach para las escalas de creencias y ansiedad superó el valor de .78, valor que evidencia una alta fiabilidad.

La Tabla 4 muestra la distribución de las frecuencias de respuesta de los docentes por país y para cada ítem o indicador empleado para evaluar las creencias hacia las matemáticas.

Tabla 4 - Distribución de las respuestas referidas a las creencias por país

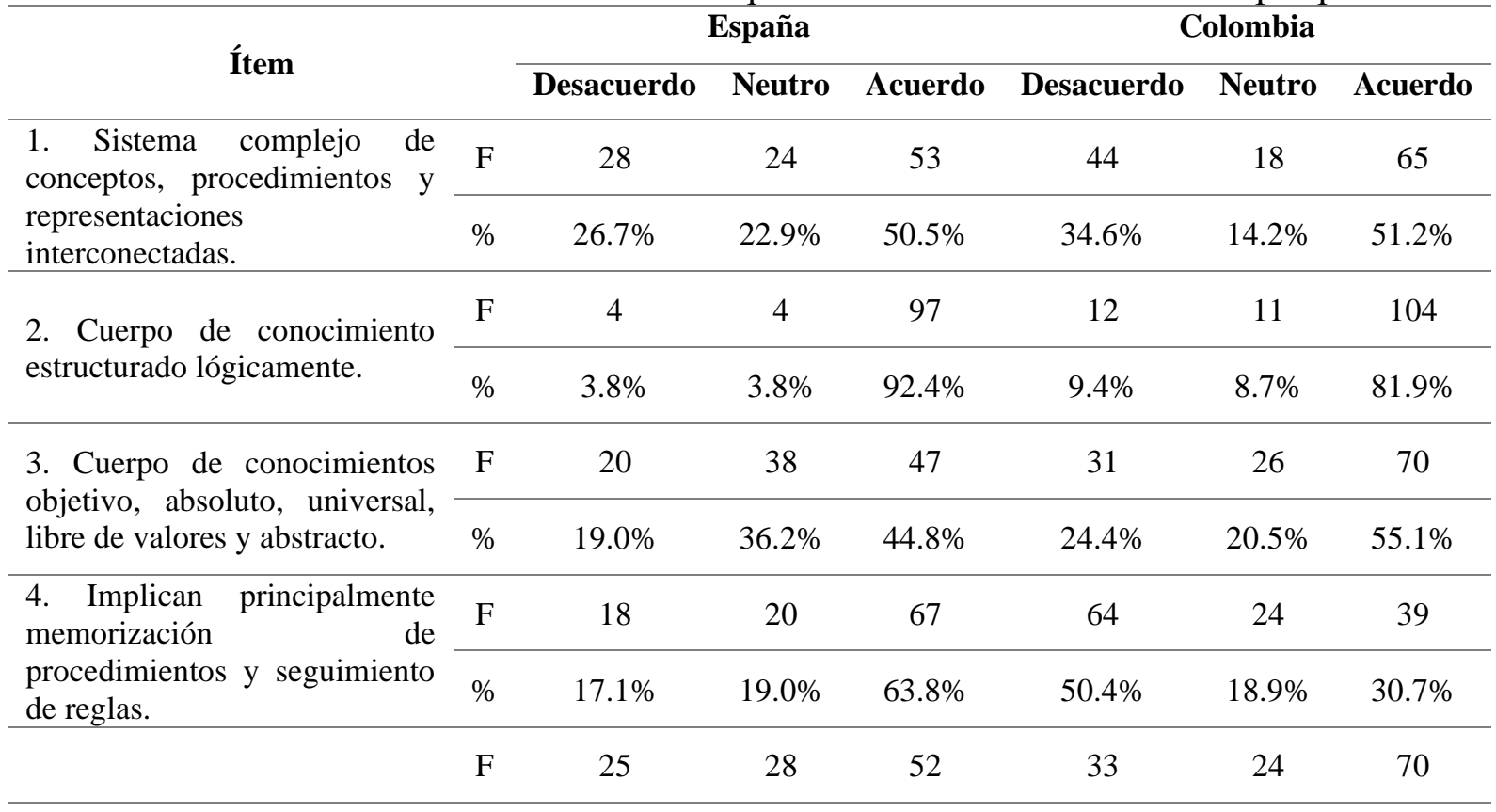




\begin{tabular}{|c|c|c|c|c|c|c|c|}
\hline $\begin{array}{l}\text { 5. El dominio de las } \\
\text { matemáticas se caracteriza por } \\
\text { la aplicación de reglas y } \\
\text { algoritmos. }\end{array}$ & $\%$ & $23.8 \%$ & $26.7 \%$ & $49.5 \%$ & $26.0 \%$ & $18.9 \%$ & $55.1 \%$ \\
\hline \multirow{2}{*}{$\begin{array}{l}\text { 6. El conocimiento matemático } \\
\text { es esencialmente fijo e } \\
\text { inmutable. }\end{array}$} & $\mathrm{F}$ & 19 & 24 & 62 & 45 & 37 & 45 \\
\hline & $\%$ & $18.1 \%$ & $22.9 \%$ & $59.0 \%$ & $35.4 \%$ & $29.1 \%$ & $35.4 \%$ \\
\hline \multirow{2}{*}{$\begin{array}{l}7 . \text { Las matemáticas están } \\
\text { abiertas a cuestionamientos, } \\
\text { argumentos o interpretaciones } \\
\text { personales }\end{array}$} & $\mathrm{F}$ & 21 & 22 & 62 & 18 & 19 & 90 \\
\hline & $\%$ & $20.0 \%$ & $21.0 \%$ & $59.0 \%$ & $14.2 \%$ & $15.0 \%$ & $70.9 \%$ \\
\hline \multirow{2}{*}{$\begin{array}{l}\text { 8. La habilidad matemática es } \\
\text { esencialmente algo con lo que } \\
\text { se nace o no se nace. }\end{array}$} & $\mathrm{F}$ & 66 & 26 & 13 & 59 & 26 & 42 \\
\hline & $\%$ & $62.9 \%$ & $24.8 \%$ & $12.4 \%$ & $46.5 \%$ & $20.5 \%$ & $33.1 \%$ \\
\hline \multirow{2}{*}{$\begin{array}{l}\text { 9. El dominio de las } \\
\text { matemáticas se materializa en } \\
\text { una manera de pensar y } \\
\text { resolver problemas. }\end{array}$} & $\mathrm{F}$ & 12 & 22 & 71 & 9 & 26 & 92 \\
\hline & $\%$ & $11.4 \%$ & $21.0 \%$ & $67.6 \%$ & $7.1 \%$ & $20.5 \%$ & $72.4 \%$ \\
\hline
\end{tabular}

Fuente: elaborada por los autores

Desde el punto de vista descriptivo, se observa que los pesos y tendencia en las respuestas son, en general, equiparables en ambos países. Sin embargo, esta equivalencia se aprecia con mayor claridad en unos indicadores que en otros. Así, por ejemplo, aproximadamente la mitad de los docentes en ambos países está de acuerdo con la afirmación de que las matemáticas representan un sistema complejo de conceptos, procedimientos y representaciones interconectadas (ítem 1). Hay un nivel de acuerdo similar (49.5\% en España; $55.1 \%$ en Colombia) al considerar que el dominio de las matemáticas se caracteriza por la aplicación de reglas y algoritmos (ítem 5). Sin embargo, se observan discrepancias entre las apreciaciones de acuerdo relacionadas con la creencia de que la habilidad matemática es esencialmente algo con lo que se nace o no se nace (ítem 8: $12.4 \%$ en España y $33.1 \%$ en Colombia).

Como se ve, el nivel de aceptación de esta afirmación en Colombia casi triplica las respuestas de los maestros españoles. También hay disparidad de respuesta en cuanto a la creencia de que las matemáticas implican principalmente memorización de procedimientos y seguimiento de reglas (ítem 4: 63.8\% España, 30.7\% Colombia), que es poco más del doble en España respecto de Colombia.

Tabla 5 - Distribución de las respuestas referidas a la ansiedad hacia las matemáticas por país

\begin{tabular}{lccccccc}
\hline \multirow{2}{*}{ Ítem } & \multicolumn{4}{c}{ España } & \multicolumn{3}{c}{ Colombia } \\
\cline { 2 - 8 } & & Desacuerdo & Neutro & Acuerdo & Desacuerdo & Neutro & Acuerdo \\
\hline \multirow{2}{*}{$\begin{array}{l}\text { 1. Es la asignatura que más me } \\
\text { gusta enseñar. }\end{array}$} & $\mathrm{F}$ & 13 & 19 & 73 & 8 & 14 & 105 \\
\cline { 2 - 8 } & $\%$ & $12.4 \%$ & $18.1 \%$ & $69.5 \%$ & $6.3 \%$ & $11.0 \%$ & $82.7 \%$ \\
\hline
\end{tabular}




\begin{tabular}{|c|c|c|c|c|c|c|c|}
\hline \multirow{2}{*}{$\begin{array}{l}\text { 2. Ha sido una asignatura difícil } \\
\text { para mí. }\end{array}$} & $\mathrm{F}$ & 26 & 13 & 66 & 78 & 22 & 27 \\
\hline & $\%$ & $24.8 \%$ & $12.4 \%$ & $62.9 \%$ & $61.4 \%$ & $17.3 \%$ & $21.3 \%$ \\
\hline \multirow{2}{*}{$\begin{array}{l}\text { 3. Es una asignatura que parece } \\
\text { difícil a mis alumnos. }\end{array}$} & $\mathrm{F}$ & 46 & 33 & 26 & 35 & 35 & 57 \\
\hline & $\%$ & $43.8 \%$ & $31.4 \%$ & $24.8 \%$ & $27.6 \%$ & $27.6 \%$ & $44.9 \%$ \\
\hline \multirow{2}{*}{$\begin{array}{l}\text { 4. Es una de las asignaturas que } \\
\text { más temía/temo. }\end{array}$} & $\mathrm{F}$ & 15 & 14 & 76 & 71 & 21 & 35 \\
\hline & $\%$ & $14.3 \%$ & $13.3 \%$ & $72.4 \%$ & $55.9 \%$ & $16.5 \%$ & $27.6 \%$ \\
\hline \multirow{2}{*}{$\begin{array}{l}\text { 5. Tengo confianza en mí } \\
\text { cuando me enfrento a un } \\
\text { problema de matemáticas. }\end{array}$} & $\mathrm{F}$ & 81 & 14 & 10 & 13 & 9 & 105 \\
\hline & $\%$ & $77.1 \%$ & $13.3 \%$ & $9.5 \%$ & $10.2 \%$ & $7.1 \%$ & $82.7 \%$ \\
\hline \multirow{2}{*}{$\begin{array}{l}\text { 6. Cuando me enfrento a un } \\
\text { problema de matemáticas me } \\
\text { siento incapaz de pensar con } \\
\text { claridad. }\end{array}$} & $\mathrm{F}$ & 7 & 14 & 84 & 93 & 11 & 23 \\
\hline & $\%$ & $6.7 \%$ & $13.3 \%$ & $80.0 \%$ & $73.2 \%$ & $8.7 \%$ & $18.1 \%$ \\
\hline \multirow{2}{*}{$\begin{array}{l}\text { 7. Estoy calmado/a y } \\
\text { tranquilo/a cuando me enfrento } \\
\text { a un problema de matemáticas. }\end{array}$} & $\mathrm{F}$ & 76 & 15 & 14 & 14 & 18 & 95 \\
\hline & $\%$ & $72.4 \%$ & $14.3 \%$ & $13.3 \%$ & $11.0 \%$ & $14.2 \%$ & $74.8 \%$ \\
\hline \multirow{2}{*}{$\begin{array}{l}\text { 8. Trabajar con las matemáticas } \\
\text { hace que me sienta muy } \\
\text { nervioso/a. }\end{array}$} & $\mathrm{F}$ & 3 & 15 & 87 & 99 & 12 & 16 \\
\hline & $\%$ & $2.9 \%$ & $14.3 \%$ & $82.9 \%$ & $78.0 \%$ & $9.4 \%$ & $12.6 \%$ \\
\hline \multirow{2}{*}{$\begin{array}{l}\text { 9. Me altero cuando tengo que } \\
\text { trabajar en problemas de } \\
\text { matemáticas. }\end{array}$} & $\mathrm{F}$ & 5 & 12 & 88 & 101 & 8 & 18 \\
\hline & $\%$ & $4.8 \%$ & $11.4 \%$ & $83.8 \%$ & $79.5 \%$ & $6.3 \%$ & $14.2 \%$ \\
\hline \multirow{2}{*}{$\begin{array}{l}\text { 10. Las matemáticas hacen que } \\
\text { me sienta incómodo/a y } \\
\text { nervioso/a. }\end{array}$} & $\mathrm{F}$ & 5 & 10 & 90 & 101 & 6 & 20 \\
\hline & $\%$ & $4.8 \%$ & $9.5 \%$ & $85.7 \%$ & $79.5 \%$ & $4.7 \%$ & $15.7 \%$ \\
\hline
\end{tabular}

Fuente: elaborada por los autores

En relación con la ansiedad hacia las matemáticas (Tabla 5) las discrepancias entre los grupos según el país aparecen más marcadas. El tema de la confianza (ítem 5) cobra relevancia, pues los niveles de acuerdo revelan importantes discrepancias entre los países comparados (9.5\% Colombia vs $82.7 \%$ España). Como es esperable, esta distribución es semejante cuando se pregunta sobre la cualidad de calma o tranquilidad cuando el encuestado se enfrenta a un problema matemático (ítem 7: 13.3\% en Colombia vs 74.8\% en España), así como también la presencia de altos niveles de nerviosismo (ítem 8: 12.6\% en Colombia vs 82.9\% en España). Las posiciones neutrales de algunas afirmaciones se mantienen en proporciones similares en ambos países, principalmente las relacionadas con la consideración de que se trata de una asignatura que parece difícil para los alumnos (ítem 3: $31.4 \%$ vs $27.6 \%$ ) y con el temor hacia la asignatura (ítem $4: 13.3 \%$ vs $16.5 \%$ ).

Al considerar la propiedad sumativa de los ítems de la escala se obtienen las 
puntuaciones totales para las subescalas de creencias y ansiedad hacia las matemáticas. La Tabla 6 muestra los estadísticos descriptivos para las puntuaciones totales en cada dimensión. Considerando la totalidad de los ítems, las creencias en las puntuaciones medias en ambos países se muestran cerradas, siendo levemente superior en España en comparación con Colombia. Sin embargo, se registra una variabilidad alta en las puntuaciones, mayor en Colombia (3.6\% en España vs $6.3 \%$ en Colombia). En referencia a la ansiedad, se reduce la discrepancia en la variabilidad, que es ahora más similar, pero aumentan las diferencias entre las medias: los docentes en España demuestran una ansiedad en más de 7 puntos por encima de la mostrada por los docentes de Colombia.

Tabla 6 - Estadísticos descriptivos para las puntuaciones totales en las escalas por país

\begin{tabular}{cccc} 
& & España & Colombia \\
\hline & Media & 31.1 & 30.8 \\
\cline { 2 - 4 } & Dt & 3.6 & 6.3 \\
\cline { 2 - 4 } $\begin{array}{c}\text { Creencias } \\
\text { sobre las } \\
\text { Matemáticas }\end{array}$ & Máx & 40.0 & 45.0 \\
\cline { 2 - 4 } & Mín & 21.0 & 17.0 \\
\cline { 2 - 4 } & Perct25 & 29.0 & 27.0 \\
\cline { 2 - 4 } & Perct75 & 33.0 & 35.0 \\
\cline { 2 - 4 } $\begin{array}{c}\text { Ansiedad } \\
\text { hacia las }\end{array}$ & Media & 35.9 & 28.2 \\
\cline { 2 - 4 } matemáticas & Máx & 4.7 & 5.8 \\
\cline { 2 - 4 } & Mín & 26.0 & 13.0 \\
\cline { 2 - 4 } & Perct25 & 32.0 & 24.0 \\
\cline { 2 - 4 } & Perct75 & 40.0 & 30.0 \\
\hline
\end{tabular}

Fuente: elaborada por los autores

Con el fin de profundizar en la evaluación de diferencias significativas de las puntuaciones totales de las escalas de creencias y ansiedad se aplicó una prueba ANOVA de un factor, considerando como factor las distintas variables que caracterizan a los docentes en la muestra (Tablas 1 y 2): país, sexo, tipo de participante, nivel de estudios, tipo de institución y de contrato.

Cuando se considera como factor el país de origen, se encuentra que las diferencias no llegan a ser significativas $\left(\mathrm{F}_{2,229}=1,548, \mathrm{p}=.067\right)$. Sin embargo, sí resultan significativas en relación con la ansiedad $\left(\mathrm{F}_{2,229}=57,703, \mathrm{p}<.000\right)$ entre los docentes de ambos paises.

Sobre la relación con el tipo de participante $\left(\mathrm{F}_{2,229}=1,417, \mathrm{p}=.24 ; \mathrm{F}_{2,229}=0.611, \mathrm{p}=.594\right)$ $y$ de institución $\left(F_{3,228}=0.067, p=.97 ; F_{3,228}=.128, p=.94\right)$ no se encontraron diferencias significativas en referencia a las creencias y a la ansiedad.

En cuanto al nivel de estudios y tipo de contrato, sí se encontraron diferencias 
significativas en los valores medios de las puntuaciones totales con referencia a la ansiedad $\left(\mathrm{F}_{4,227}=.93, \mathrm{p}<.00 ; \mathrm{F}_{2,229}=3.43, \mathrm{p}=.03\right)$. Un análisis post hoc de subconjuntos homogéneos de Duncan al nivel del 5\% indica, por una parte, que los docentes que tienen un nivel de estudios de maestría o doctorados presentan una puntuación media significativamente menor de los que tienen una diplomatura o licenciatura. Por otra parte, la puntuación media de la ansiedad de quienes tienen un contrato temporal es significativamente más alta en comparación con la que manifiestan los docentes que tiene contrataciones permanentes.

Otra perspectiva para analizar el sistema de creencias hacia las matemáticas se basa en la consideración de las distintas posturas epistémicas asumidas por los informantes. Así, de acuerdo con León-Mantero et al. (2020) y con García-Moya et al. (2020), los ítems 1-3 hacen referencia al euclidianismo, los ítems 4 al 6 al cuasi-empirismo y los ítems 7 al 9 al constructivismo.

En relación con lo anterior, la Tabla 7 muestra los estadísticos descriptivos de acuerdo con las posturas epistemológicas planteadas en el sistema de creencias. El euclidianismo presenta una media superior (10.9) en comparación con el constructivismo (10.1) y el cuasiempirismo (9.9). La mayor variación se presenta en el cuasi-empirismo, con una desviación estándar aproximada de tres puntos mientras que las otras dos están por debajo de este valor en al menos cinco décimas.

Tabla 7 - Estadísticos descriptivos para las puntuaciones de acuerdo con las posturas epistemológicas del sistema de creencias

\begin{tabular}{ccccccc}
\hline Posturas Epistemológicas & Media & Dt & Máx & Mín & Perct25 & Perct75 \\
\hline Euclidianismo & 10.9 & 2.5 & 15.0 & 3.0 & 9.0 & 13.0 \\
\hline Cuasi-empirismo & 9.9 & 2.9 & 15.0 & 3.0 & 7.0 & 12.0 \\
\hline Constructivismo & 10.1 & 2.1 & 15.0 & 3.0 & 9.0 & 11.0 \\
\hline \multicolumn{7}{c}{ Fuente: elaborada por los autores }
\end{tabular}

Al considerar los estilos predominantes en los maestros según su país de origen (Tabla 8) se evidencian tendencias similares en cuanto al euclidianismo (18.1\% vs $19.4 \%$ ). Sin embargo, en relación al cuasi-empirismo se observa una tendencia más marcada entre docentes españoles (15.1\%) en comparación con los colombianos, situación que se invierte cuando comparamos el constructivismo, en el cual la proporción de docentes colombianos es claramente superior a la de los españoles (11.2\% vs 2.6\%). En una proporción aproximada del $10 \%$ en cada grupo de docentes se presenta una combinación de estilos epistémicos, en el que dos o las tres posturas analizadas alcanzan puntuaciones significativas y similares entre sí.

Tabla 8 - Distribución de posturas epistémicas por país

\begin{tabular}{lcccccc}
\hline \multirow{2}{*}{ Postura epistemológica } & \multicolumn{2}{c}{ España } & \multicolumn{2}{c}{ Colombia } & \multicolumn{2}{c}{ Total } \\
\cline { 2 - 7 } & $\mathrm{F}$ & $\%$ & $\mathrm{~F}$ & $\%$ & $\mathrm{~F}$ & $\%$ \\
\hline Sin postura clara & 1 & $0.4 \%$ & 13 & $5.6 \%$ & 14 & $6.0 \%$ \\
\hline
\end{tabular}




\begin{tabular}{lcccccc}
\hline Euclidianismo & 42 & $18.1 \%$ & 45 & $19.4 \%$ & 87 & $37.5 \%$ \\
\hline Cuasi-empirismo & 35 & $15.1 \%$ & 16 & $6.9 \%$ & 51 & $22.0 \%$ \\
\hline Constructivismo & 6 & $2.6 \%$ & 26 & $11.2 \%$ & 32 & $13.8 \%$ \\
\hline Combinación de posturas & 21 & $9.1 \%$ & 27 & $11.6 \%$ & 48 & $20.7 \%$ \\
\hline
\end{tabular}

Fuente: elaborada por los autores

Al valorar una posible relación de dependencia entre la postura epistémica y el país de origen encontramos un valor de chi-cuadrado de 28.89 con un p-valor asociado de 0.00 que permite rechazar la hipótesis de independencia y concluir que existe una asociación clara entre los estilos epistemológicos del sistema de creencias hacia las matemáticas y el país de origen del docente.

Otra relación que se ha mostrado interesante consiste en el nivel de correlación existente entre las puntuaciones en la escala de ansiedad y las distintas posturas epistémicas. Un valor de $\mathrm{r}$ de $-0.394(p<.000)$ indica una relación moderada entre el cuasi-empirismo y la ansiedad hacia las matemáticas y prácticamente nula con las otras posturas epistémicas $(\mathrm{r}=0.07, p=.30 ; \mathrm{r}=-$ $0.07, p=.27)$.

\section{Discusión y conclusiones}

Este estudio pretendía validar el instrumento empleado mediante la Teoría Clásica de los Test evaluando su fiabilidad, validez y sensibilidad cultural, así como explorar las posibles similitudes y diferencias sobre creencias y ansiedad hacia las matemáticas de los docentes y futuros docentes en España y Colombia.

En cuanto al instrumento utilizado, se partió de dos cuestionarios mencionados en los antecedentes y se construyó el de esta investigación. En el proceso de validación se determinó la capacidad discriminativa de cada ítem, reportando su aporte a la subescala que pertenece y a la escala global. Esto generó algunos ajustes que se complementaron con el análisis de la sensibilidad cultural derivada del juicio de expertos. Finalmente, en cuanto a la estructura del cuestionario, se tomó como criterio el coeficiente de confiabilidad alfa de Cronbach dadas las características de la opción de respuesta adoptada.

En lo que respecta al estudio comparativo, los resultados presentados y sus respectivas interpretaciones se ajustan a las características propias de una investigación descriptiva; los resultados evidencian distintos grados de similitud y diferencia en lo que respecta al conjunto de creencias y ansiedad que manifiestan los docentes hacia las matemáticas en función de los factores analizados. Por ejemplo, al considerar el país de origen, los resultados demuestran que los docentes españoles consideran en mayor proporción las matemáticas como un sistema 
complejo que funciona bajo reglas y algoritmos, que la que se detecta entre los docentes colombianos. Sin embargo, al determinar las puntuaciones totales se revela un ajuste en el conjunto de indicadores que permite apreciar que los docentes de ambos países tienen una visión similar del sistema de creencias en función de los indicadores abordados. Esto quiere decir que ambos grupos de informantes consideran que las matemáticas son un cuerpo sólido de conocimientos que obedece a reglas y procedimientos, lo que requiere del desarrollo de habilidades particulares para abordarlo.

Por otra parte, en referencia a la ansiedad experimentada, los resultados descubren varias divergencias: en primer lugar, los docentes españoles mostraron mayores niveles de ansiedad a través de comportamientos de nerviosismo, tensión o temor hacia las matemáticas. Sin embargo, es de hacer notar que, en línea con otras investigaciones realizadas sobre ansiedad en este colectivo (HADLEY; DORWARD, 2011; UYSAL; DEDE, 2016), el nivel de ansiedad general no resultó alto. Sin embargo, cuando se establecen las comparaciones se demuestra la existencia de un componente de aversión hacia la asignatura, mayor en el docente español que en el colombiano, así como, también en este último un mayor nivel de confianza cuando se enfrenta a la resolución de un problema matemático.

Un factor concreto y explicativo de estas diferencias podría estar representado por la condición de los participantes, pues la gran mayoría de los docentes colombianos han alcanzado su grado de licenciatura recientemente, por lo cual mantienen sus conocimientos académicos y pedagógicos recientes y presentan un dominio conceptual y procedimental superior. En línea con lo anterior, estos hallazgos se relacionan con los encontrados en investigaciones como la de Hadley y Dorward (2011), quienes argumentan que existe una correlación entre la ansiedad manifestada por los docentes hacia las matemáticas y la experimentada respecto de su práctica docente, y que esta ansiedad se va moderando a partir de su experiencia y continuo ejercicio profesional, resultado hallado también por León-Mantero et al. (2020), quienes encuentran ansiedad más alta en profesorado con experiencia media que en aquellos con poca o dilatada experiencia.

Entre el conjunto de factores encontrados que pueden marcar estas diferencias se encuentra el lugar de origen, lo cual resultaría atribuible a las diferencias en los sistemas escolares de cada país y en la formación inicial de los docentes. Autores como Else-Quest, Hyde y Linn (2010) y Frenzel, Pekrun y Goetz (2007) destacan que la diferencia en la formación de los maestros en distintos países establece marcados contrastes relativos al género, roles sociales e influencias culturales que pueden influir en la práctica docente.

Por su parte, otros investigadores, entre los que se destaca a Uysal y Dede (2016), 
señalan que las creencias de los docentes hacia las matemáticas juegan un papel determinante en el rendimiento de sus alumnos y en la formación de las creencias y en la ansiedad de estos hacia las matemáticas. En consecuencia, reconocer la influencia de las creencias del docente en el desarrollo del sistema de creencias de los estudiantes sería un punto importante para los actuales maestros. Además, les podría ayudar a emplear unas estrategias pedagógicas que le permitieran minimizar su ansiedad hacia las matemáticas, $y$, de igual manera, no generarla en sus estudiantes. Este aspecto no se ha estudiado en este trabajo, y constituiría una de sus posibles ampliaciones a futuro.

Otro factor significativo vinculado a la ansiedad resultó ser el nivel de estudios y el tipo de contratación del docente en ejercicio. Los docentes con postgrado se muestran más conservadores en relación con el sistema de creencias en comparación con los que solo han cursado una diplomatura o licenciatura. Asimismo, las condiciones laborales bajo contratos temporales, bien sea por la formación recibida, o la ausencia de programas de capacitación, marcan, de alguna manera, la ansiedad de los docentes hacia las matemáticas durante su práctica.

Orozco-Moret y Ángel-Díaz (2009) señalan que los docentes motivados por distintas razones, entre las que se incluye niveles superiores de estudios y mayor estabilidad laboral, se muestran más satisfechos en el ejercicio de la práctica profesional, lo cual incide en la transmisión de los contenidos disciplinares con menor ansiedad, lo que es percibido por los alumnos. Por otra parte, la relación que se evidencia entre la formación recibida y la ansiedad está en línea con los hallazgos teóricos de Haciomeroglu (2013) y Swars et al. (2009), quienes expresan que, a mayor formación, los docentes experimentan menos ansiedad durante sus prácticas.

En relación con las posturas epistémicas, resultó significativo el país de origen del docente, destacándose una tendencia clara de los docentes españoles hacia el cuasi-empirismo y de los colombianos hacia el constructivismo. En cuanto al euclidianismo, no se evidenciaron diferencias significativas entre ambos países. Sin embargo, resalta una correlación moderadamente negativa entre quienes asumen una postura cuasi-empirista y la ansiedad experimentada hacia las matemáticas, que fue también hallada por León-Mantero et al. (2020) en maestros españoles. Podría interpretarse como que aquellos que perciben las matemáticas como medio para explicar los fenómenos que ocurren a su alrededor se sintieran menos ansiosos ante su enseñanza, pues perciben su utilidad, y su estudio como una necesidad natural.

Dadas las anteriores reflexiones conviene señalar que, a pesar de la relevancia de los hallazgos, la elección de la muestra de conveniencia limita la validez de las conclusiones. Por 
ello, sería necesario ampliar la misma de manera que fuera más representativa de los colectivos estudiados. También sería necesario profundizar sobre el conjunto de situaciones que inciden en las creencias y la ansiedad en la enseñanza de las matemáticas en docentes de educación básica, y determinar en qué medida estas influyen en la práctica de estos docentes de matemáticas.

Por lo anterior, conviene estudiar no solo la relación entre ansiedad y creencias, sino también en relación con otros elementos presentes en la práctica docente, tales como las estrategias de resolución de problemas, la representación y la comunicación en el aula, todo ello con el fin de lograr una aproximación al complejo compendio que configura la idoneidad de la acción docente en el aula.

\section{Agradecimientos}

Proyecto desarrollado bajo el auspicio de la Secretaría Técnica del Área de Cooperación Científica de la Organización de Estados Iberoamericanos para la Educación, la Ciencia y la Cultura, a cargo de Juan Carlos Toscano (2016-2018), con el apoyo de la Universidad de Castilla-La Mancha, España y la Universidad Francisco de Paula Santander, Cúcuta - Colombia (Grupo de Investigación en Pedagogía y Practicas Pedagógicas - GIPEPP).

\section{Referencias}

AIKEN, L. R. Update on attitudes and other affective variables in learning mathematics. Review of Educational Research, Washington DC, v. 46, n. 2, p. 293-311, 1976.

ANDREWS, P.; XENOFONTOS, C. Analysing the relationship between the problem-solving-related beliefs, competence and teaching of three Cypriot primary teachers. Journal of Mathematics Teacher Education, Cham, v. 18, n. 4, p. 299-325, 2015.

ASSOR, A.; KAPPLAN, H.; KANAT-MAYMON, Y.; ROTH, G. Directly controlling teacher behaviors as predictors of poor motivation an engagement in girls and boys: The role on anger and anxiety. Learning and Instruction, London, v. 15, n. 5, p. 397-413, 2005.

AUZMENDI, E. Las actitudes hacia la matemática-estadística en las enseñanzas medias y universitaria: Características y medición. Bilbao: Ediciones Mensajero, 1992.

BAROODY, A. J.; COSLICK, R. T. Fostering Children's Mathematical Power: An Investigative Approach to K-8 Mathematics Instruction. Mahwah: Lawrence Erlbaum, 1998.

BISHOP A. J.; NICKSON M. A Review of Research in Mathematical Education. Part B. London: NFER Nelson Publishing Co Ltd, 1983.

BRÍGIDO, M.; BERMEJO, M. L.; CONDE, C.; BORRACHERO, A. B.; MELLADO, V. Estudio longitudinal de las emociones en ciencias de estudiantes de maestro. Revista Galego-Portuguesa de 
Psicoloxía e Educación, La Coruña, v. 18, n. 2, p. 161- 179, 2010.

CARMONA, J. Una revisión de las evidencias de fiabilidad y validez de los cuestionarios de actitudes y ansiedad hacia la estadística. Statistics Education Research Journal, Auckland, v. 3, n. 1, p. 5-28, 2004.

CASIS, M.; RICO, N.; CASTRO, E. Motivación, autoconfianza y ansiedad como descriptores de la actitud hacia las matemáticas de los futuros profesores de educación básica de Chile. PNA, Granada, v. 11, n. 3, p. 181-203, 2017.

COHEN, R. J.; SWERDLIK M. E. Psychological Testing and Assessment: An Introduction to Tests and Measurement. New York City: McGrawHill, 2010.

DOGAN, H. Emotion, confidence, perception and expectation. Case of mathematics. International Journal of Science and Mathematics Education, Taipei, v. 10, p. 49-69, 2012.

ELSE-QUEST, N. M.; HYDE, J. S.; LINN, M. C. Cross-national patterns of gender differences in mathematics: a meta-analysis. Psychological bulletin, Washington DC, v. 136, n. 1, p. 103-127, 2010.

ERNEST P. Philosophy, mathematics and education. International Journal of Education, Science and Technology, London, v. 20, n. 4, p. 555-559, 1989.

ESTRADA, A.; BAZÁN, J.L.; APARICIO, A. Un estudio comparativo de las actitudes hacia la estadística en profesores españoles y peruanos. Revista Iberoamericana de Educación Matemática, São Paulo, v. 24, p. 45-56, 2010.

ESTRADA. A. Actitudes hacia la estadística: un estudio con profesores de educación primaria en formación y en ejercicio. En: CAMACHO, M.; FLORES, P.; BOLEA, P. (ed.). Actas del XI Simposio de la SEIEM. Santa Cruz de Tenerife: Sociedad Española de Investigación en Educación Matemática, 2007. p. 121-140.

FERNÁNDEZ, R.; HERNÁNDEZ, C. A.; PRADA, R.; RAMÍREZ, P. Dominio afectivo y prácticas pedagógicas de docentes de Matemáticas: Un estudio de revisión. Revista Espacios, Caracas, v. 39, n. 23, p. 25-35, 2018.

FERNÁNDEZ-ABASCAL, E.; JIMÉNEZ, M. P.; MARTÍN, M. D. Emoción y motivación: La adaptación humana. Madrid: Editorial Universitaria Ramón Areces, 2003.

FERNÁNDEZ-CÉZAR, R.; AGUIRRE-PÉREZ, C. Actitudes iniciales hacia las matemáticas de los alumnos de grado de magisterio de Educación Primaria: Estudio de una situación en el EEES. Unión, São Paulo, v. 23, p. 107-116, 2010a.

FERNÁNDEZ-CÉZAR, R.; AGUIRRE-PÉREZ, C. Evolution of the attitudes towards Mathematics of Spanish students of Primary Education Degree. International Journal for Knowledge, Science and Technology, Bilbao, v. 2, p. 14-19, 2010 b.

FERNÁNDEZ-CÉZAR, R.; SOLANO-PINTO, N.; RIZZO, K.; GOMEZESCOBAR-CAMINO, A.; IGLESIAS, L. M.; ESPINOSA, A. Las actitudes hacia las matemáticas en estudiantes y maestros de educación infantil y primaria: revisión de la adecuación de una escala para su medida. Revista Iberoamericana de Ciencia, Tecnología y Sociedad-CTS, Buenos Aires, v. 11, n. 33, p. 227-238, 2016.

FERNÁNDEZ-CÉZAR, R.; PRADA-NÚÑEZ, R.; SOLANO-PINTO, N. Beliefs towards Mathematics in Elementary Education Teachers: a comparative study. Journal of Research in Science, Mathematics and Technology Education, Almaty, v. 1, n. 3, p. 329-345, 2018. 
FERNANDEZ-CÉZAR, R.; PRADA-NÚÑEZ, R.; HERNÁNDEZ-SUÁREZ, C. A. Procesos matemáticos en la práctica pedagógica: un comparativo entre Colombia y España. Aibi Revista de Investigación, Administración e Ingenierías, Cúcuta, v. 8, n. 1, p. 29-36, 2020.

FIVES, H.; LACATENA, N.; GERARD, L. Teachers' beliefs about teaching (and learning). In: FIVES, H.; GILL, M. G. (ed.). International handbook of research on teachers' beliefs. New York: Routledge, 2014, p. 249-265.

FRENZEL, A. C.; PEKRUN, R.; GOETZ, T. Perceived learning environment and students' emotional experiences: A multilevel analysis of mathematics classrooms. Learning and Instruction, London, v. 17, n. 5 , p. $478-493,2007$.

GARCÍA-MOYA, M.; GÓMEZ-ESCOBAR, A.; SOLANO-PINTO, N.; FERNÁNDEZ-CÉZAR, R. Las creencias de los futuros maestros sobre la enseñanza y aprendizaje de las matemáticas. Revista Espacios, Caracas, v. 41, n. 9, p. 1-14, 2020.

GARCÍA, M.; MARTÍNEZ, G. The history of a teacher's relief of his mathematics anxiety: the case of Diego. Educational Studies in Mathematics, Cham, v. 103, n. 3, p. 273-291, 2020.

GASCÓN, J. Incidencia del modelo epistemológico de las matemáticas sobre las prácticas docentes. Revista latinoamericana de Investigación matemática educativa, Ciudad de México, v. 4, n. 2, p. 129-159, 2001.

GODINO, J.; BATANERO, C.; FONT, V. Fundamentos de la Enseñanza y el Aprendizaje de las Matemáticas para maestros. Granada: Universidad de Granada, 2003.

GODINO, J.; BATANERO, C.; FONT, V. Fundamentos de la Enseñanza y el Aprendizaje de las Matemáticas. En: GODINO, J. (Dir.). Didáctica de las Matemáticas para Maestros. Granada: Universidad de Granada, 2004. p. 5-153.

GOLDIN, G.; RÖSKEN, B.; TÖRNER, G. Beliefs-no longer a hidden variable in mathematical teaching and learning processes. In: MAAB, J.; SCHLOGLMANN, W. (ed.). Beliefs and attitudes in mathematics education: New research results. Rotterdam: Sense publishers, 2009. p. 9-28.

GÓMEZ, I. Matemática emocional: Los afectos en el aprendizaje matemático. Madrid: Narcea, 2000.

GÓMEZ-CHACÓN, I. M. La tarea intelectual en matemáticas afecto, meta-afecto y los sistemas de creencias. Boletín de la asociación matemática venezolana, Caracas, v. 10, n. 2, p. 225-247, 2003.

GÓMEZESCOBAR, A; FERNÁNDEZ, R. Las actitudes hacia las matemáticas de maestros de educación infantil y primaria. En: JORNADAS CIENTÍFICAS DE DIFUSIÓN DE LA INVESTIGACIÓN, 9., 2016, Cuenca. Actas... Cuenca: Universidad de Castilla La Mancha, 2016. p. $1-11$.

HACIOMEROGLU, G. The Field Experiences of Student Teachers and Effective Mathematics Teaching in Turkey. Australian Journal of Teacher Education, Perth, v. 38, n. 2, p. 132-142, 2013.

HADLEY, K. M.; DORWARD, J. The Relationship among Elementary Teachers' Mathematics Anxiety, Mathematics Instructional Practices, and Student Mathematics Achievement. Journal of Curriculum and Instruction, Greenville, v. 5, n. 2, p. 27-44, 2011.

HANNULA, M. S.; DI MARTINO, P.; PANTZIARA, M.; ZHANG, Q.; MORSELLI, F.; HEYDMETZUYANIM, E.; LUTOVAC, S.; KAASILA, R.; MIDDLETON, J.A.; JANSEN, A.; GOLDIN, G.A. Attitudes, Beliefs, Motivation and Identity in Mathematics Education: An Overview of the Field and Future Directions. Hamburg: ICME-13 Topical Surveys - Springer Open, 2016. 
HANNULA, M. S.; LEDER, G. C.; MORSELLI, F.; VOLLSTEDT, M.; ZHANG, Q. (ed.) Affect and Mathematics Education. Fresh Perspectives on Motivation, Engagement, and Identity. Cham: Springer, 2019.

INGRAM, N.; HATISARU, V.; GROOTENBOER, P.; BESWICK, K. Researching the Affective Domain in Mathematics Education. In: WAY, J.; ATTARD, C.; ANDERSON, J.; BOBIS, J.; MCMASTER, H.; CARTWRIGHT, K. (ed.). Research in Mathematics Education in Australasia 2016-2019. Singapore: Springer, 2020. p. 147-175.

JANKVIST, U. T.; VAN DEN HEUVEL-PANHUIZEN, M.; VELDHUIS, M. (ed.). Proceedings of the Eleventh Congress of the European Society for Research in Mathematics Education. Utrecht: Freudenthal Group \& Freudenthal Institute, Utrecht University and ERME, 2019.

JONG, C.; HODGES, T. E.; ROYAL, K. D.; WELDER, R. M. Instruments to Measure Elementary Preservice Teachers' Conceptions. Educational Research Quarterly, Grambling, v. 39, n. 1, p. 21-48, 2015.

KOBALLA, T. R.; CRAWLEY, F. E. The influence of attitude on science teaching and learningand learning. School Science and Mathematics, Hoboken, v. 85, n. 3, p. 222-232, 1985.

LAKATOS, I. Matemáticas, ciencia y epistemología. Madrid: Alianza Editorial, 1978.

LEÓN-MANTERO, C.; SOLANO-PINTO, N. S.; GÓMEZESCOBAR, A.; FERNÁNDEZ-CÉZAR, R. Dominio afectivo y prácticas docentes en Educación Matemática: un estudio exploratorio en maestros. Unión, São Paulo, v. 16, n. 58, p. 129-149, 2020.

LERA, C.; DEULOFEU, J. Conocimientos y creencias entorno a la resolución de problemas de profesores y estudiantes de profesor de matemáticas. Bolema, Rio Claro, v. 28, n. 48, p. 191-208, abr, 2014.

LERA, C.; DEULOFEU, J. Creencias de profesores y estudiantes de profesor de educación primaria y secundaria sobre los problemas de matemáticas. REDIMAT, Barcelona, v. 4, n. 2, p. 161-178, 2015.

LERMAN, S. (ed.). Encyclopedia of mathematics education. Dordrecht: Springer Netherlands, 2020.

COLOMBIA. Ley $\mathbf{n}^{\circ} 115$ de 8 de febrero de 1994. Por el cual se expide la Ley General de Educación. Diario Oficial de Colombia. Bogotá, Colombia, 1994. Disponible en: http://www.secretariasenado.gov.co/senado/basedoc/ley_0115_1994.html Acceso en: 26 oct. 2020.

ESPAÑA. Ley Orgánica $\mathbf{n}^{\circ} \mathbf{2}$ de Educación de 3 de mayo de 2006. Boletín Oficial. Madrid, España, 2006. Disponible en https://www.boe.es/buscar/pdf/2006/BOE-A-2006-7899-consolidado.pdf Acceso en: 26 oct. 2020

LLINARES, S. Conocimiento profesional del profesor de matemáticas: conocimiento, creencias y contexto en relación a la noción de función. Desenvolvimento profissional dos professores de matemática. En: PONTE, J. P.; MONTEIRO, C.; MAIA, M.; SERRAZINA, L.; LOURERIO, C. (coord.). Desenvolvimiento Profesional dos Profesores de matemática: Que formação. Lisboa: Sociedade Portuguesa de Ciencias da Educaçao, 1996. p. 47-82.

MARTÍNEZ, O. Dominio afectivo en educación matemática. Paradigma, Maracay, v. 26, n. 2, p. 7 34, 2005.

MARTÍNEZ-ARTERO, R.; NORTES, A. Actitud hacia la matemática en futuros docentes de primaria y de secundaria. Edetania, Valencia, v. 44, p. 47-76, 2013. 
MARTÍNEZ-ARTERO, R.; NORTES, A. ¿Tienen ansiedad hacia las matemáticas los futuros matemáticos? Profesorado, Granada, v. 18, n. 2, p. 153-170, 2014.

MAZ-MACHADO, A.; LEÓN-MANTERO, C.; CASAS, J. C.; RENAUDO, J. A. Attitude towards mathematics of computer engineering students. British Journal of Education, Society y Behavioural Science, Hooghly, v. 8, n. 2, p. 127-133, 2015.

MÉNDEZ, I. Prácticas docentes y rendimiento estudiantil. Evidencia a partir de TALIS 2013 y PISA. Logroño: Fundación Santillana, 2015.

MUÑIZ, J.; FIDALGO, A. M.; GARCÍA-CUETO, E.; MARTÍNEZ, R.; MORENO, R. Análisis de ítems. Madrid: La Muralla, 2005.

OP'T EYNDE, P.; DE CORTE, E.; VERSCHAFFEL, L. Framing students' mathematics-related beliefs, In: LEDER, G. C.; PEHKONEN, E.; TÖRNER, G. (ed.). Beliefs: A hidden variable in mathematics education? Mathematics Education Library. New York: Kluwer Academic, 2003. p. 1338.

OROZCO-MORET, C.; ÁNGEL-DÍAZ, M. Formación del razonamiento lógico-matemático. Interciencia, Caracas, v. 39, n. 4, p. 630-636, sep. 2009.

OTTMAR, E. R.; RIMM-KAUFMAN, S. E.; BERRY, R. Q.; LARSEN, R. A. Does the Responsive Classroom approach affect the use of standards-based mathematics teaching practices? Results from a randomized controlled trial. The Elementary School Journal, Chicago, v. 113, n. 3, p. 434-457, 2013.

PALACIOS, A.; ARIAS, V.; ARIAS, B. Las actitudes hacia las matemáticas: construcción y validación de un instrumento para su medida. Revista de Psicodidáctica, Bilbao, v. 19, n. 1, p. 67-91, 2014.

PALACIOS, A.; HIDALGO, S.; MAROTO, A.; ORTEGA, T. Causas y consecuencias de la ansiedad matemática mediante un modelo de ecuaciones estructurales. Enseñanza de las ciencias, Barcelona, v. 31, n. 2, p. 93-111, 2013.

PEHKONEN, E.; PIETILÄ, A. On Relationships between beliefs and knowledge in Mathematics Education. In: CONFERENCE OF THE EUROPEAN SOCIETY FOR RESEARCH IN MATHEMATICS EDUCATION, 3., 2003, Bellaria. Proceedings...Bellaria: Department of Mathematics of the University of Pisa, 2003. p. 1-8.

PENDERGAST, E.; LIEBERMAN-BETZ, R. G.; VAIL, C. O. Attitudes and beliefs of prekindergarten teachers toward teaching science to young children. Early Childhood Education Journal, Cham, v. 45, n. 1, p. $43-52,2017$.

PÉREZ, S.; GUILLÉN, G. Estudio exploratorio sobre creencias y concepciones de profesores de secundaria en relación con la geometría y su enseñanza. En: CAMACHO, M.; FLORES, P.; BOLEA, M. P. (Eds.) Investigación en educación matemática: comunicaciones de los grupos de investigación del XI Simposio de la SEIEM, San Cristóbal de la Laguna, Tenerife: Sociedad Española de Investigación en Educación Matemática, 2007. p. 295-306

PÉREZ-TYTECA, P.; CASTRO, E.; RICO, L.; CASTRO, E. Ansiedad matemática, género y ramas de conocimiento en alumnos universitarios. Enseñanza de las ciencias, Barcelona, v. 29, n. 2, p. 237250, 2011.

PRADA, R.; HERNÁNDEZ, C.; RAMÍREZ, P. Enseñanza del cálculo diferencial: Un análisis de las dificultades en estudiantes universitarios. Cúcuta/Bogotá: Universidad Francisco de Paula Santander/ Ecoe Ediciones, 2020. 
PRIETO, G.; DELGADO, A. Fiabilidad y validez. Papeles del psicólogo, Madrid, v. 31, n. 1, p. 6774, 2010.

RAMOS, A. B. Objetos personales matemáticos y didácticos del profesorado y cambios institucionales: El caso de la contextualización de las funciones en una facultad de ciencias económicas y sociales. 2005. Tesis (Doctorado en Ciències Experimentals i la Matemàtica) Universidad de Barcelona, Barcelona, 2005.

REYES-RODRÍGUEZ, A. V.; RONDERO-GUERRERO, C.; ACOSTA-HERNÁNDEZ, J. A.; CAMPOS-NAVA, M.; TORRES-RODRÍGUEZ, A. A. Reduccionismo Didáctico y Creencias de Profesores acerca del Teorema de Pitágoras. Bolema, Rio Claro, v. 31, n. 59, p. 968-983, 2017. DOI: http://dx.doi.org/10.1590/1980-4415v31n59a06.

RODRÍGUEZ-MANTILLA, J. M.; FERNÁNDEZ-CRUZ, F. J. Identificación de perfiles de estudiantes en los alumnos españoles participantes en PISA 2015: Variables familiares, sociales y de motivación hacia el logro. En: CONGRESO INTERNACIONAL DE INVESTIGACIÓN EDUCATIVA, INTERDISCIPLINARIEDAD Y TRANSFERENCIA, 18., 2017, Salamanca. Actas... Salamanca: Universidad de Salamanca, 2017. p. 1-11.

RODRIGUES, M.; FERNÁNDEZ, R.; ROSA, J. Attitudes towards mathematics in pre-service teachers: a comparative study between Spain and Portugal focusing on anxiety. Revista Internacional de Pesquisa em Educação Matemática, Brasilia, v. 7, n. 2, p. 71-87, 2017.

SÁNCHEZ, J.; SEGOVIA, I.; MIÑÁN, A. Exploración de la ansiedad hacia las matemáticas en los futuros maestros de educación primaria. Revista de curriculum y formación del profesorado, Granada, v. 15, n. 3, p. 297-312, 2011.

SANDERS PEIRCE, C. La fijación de la creencia. Cómo aclarar nuestras ideas. Traducción de Lorena Villamil García. Oviedo: KRK Ediciones, 2007.

SAYERS, J. Primary teachers' attitudes towards and beliefs about mathematics teaching: the collective culture of one English primary school. In: CONFERENCE OF THE EUROPEAN SOCIETY FOR RESEARCH IN MATHEMATICS EDUCATION (CERME), 5., 2007, Larnaca. Proceedings... Larnaca: University of Cyprus and ERME, 2007. p. 1984-1993.

SKOTT, J. The promises, problems, and prospects of research on teachers' beliefs. In: FIVES, H.; GILL, M. G. (ed.). International handbook of research on teachers' beliefs. New York: Routledge, 2014. p. 13-30.

SIRECI, S. G. The construct of content validity. In: Zumbo, B. D. (ed.). Validity Theory and the Methods Used in Validation: Perspectives from the Social and Behavioral Sciences. Dordrecht: Kluwer Academic Press, 1998. p. 83-117.

SIVUNEN, M.; PEHKONEN, E. Finnish Elementary Teachers' Conceptions on Problem Solving in Mathematics Education. In: MAAB, J.; SCHLOGLMANN, W. (ed.). Beliefs and attitudes in mathematics education: New research results. Rotterdam: Sense publishers, 2009. p. 75-86.

SUINN, R. M.; WINSTON, E. H. The mathematics anxiety rating scale, a brief version: psychometric data. Psychological reports, London, v. 92, n. 1, p. 167-173, 2003.

SWARS, S. L.; SMITH, S. Z.; SMITH, M. E.; HART, L. C. A longitudinal study of effects of a developmental teacher preparation program on elementary prospective teachers' mathematical beliefs. Journal of Mathematics Teacher Education, Cham, v. 12, n. 1, p. 47-66, 2009.

SWEETING, K. Early years teachers' attitudes towards mathematics. 2011. Thesis (Masters of Education) - University of Technology, Queensland, 2011. 
THIEL, O. Teachers' attitudes towards mathematics in early childhood education. European Early

Childhood Education Research Journal, London, v. 18, n. 1, p. 105-115, 2010.

THOMPSON, A. Teacher's Beliefs and Conceptions: A Synthesis of the Research. In: GROUWS, D. A. (ed.). Handbook of Research on Mathematics Teaching and Learning. New York: Macmillan, 1992. p. 127-146.

UYSAL, F.; DEDE, Y. Mathematics Anxiety and Beliefs of Turkish Pre-service Elementary Teachers. Eurasia, Derbyshire, v. 12, n. 8, p. 2171-2186, 2016.

VILA, A.; CALLEJO, M. L. Matemáticas para aprender a pensar: el papel de las creencias en la resolución de problemas. Madrid: Narcea, 2004.

WOOD, E. F. Math anxiety and elementary teachers: What does research tell us? For the learning of mathematics, New Westminster, v. 8, n. 1, p. 8-13, 1988.

Submetido em 31 de Janeiro de 2019. Aprovado em 11 de Junho de 2020. 